Pure Appl. Chem., Vol. 81, No. 1, pp. 19-43, 2009.

doi:10.1351/PAC-CON-08-10-18

(C) 2009 IUPAC

\title{
Magnetic field effects in chemical systems*
}

\author{
Christopher T. Rodgers ${ }^{\ddagger}$ \\ Oxford Centre for Clinical Magnetic Resonance Research, University of Oxford, \\ John Radcliffe Hospital, Oxford, OX3 9DU, UK
}

\begin{abstract}
Chemical reactions that involve radical intermediates can be influenced by magnetic fields, which act to alter their rate, yield, or product distribution. These effects have been studied extensively in liquids, solids, and constrained media such as micelles. They may be interpreted using the radical pair mechanism (RPM). Such effects are central to the field of spin chemistry of which there have been several detailed and extensive reviews. This review instead presents an introductory account of the field of spin chemistry, suitable for use by graduate students or researchers who are new to the area. It proceeds by giving a brief historical overview of the development of spin chemistry, before introducing the essential theory. This is then illustrated by application to a series of recent developments in solution-phase magnetic field effects (MFEs). The closing pages of this review describe the role played by spin chemistry in the remarkable magnetic compass sense of birds and other animals.
\end{abstract}

Keywords: spin chemistry; radical pair; RPM; magnetic; magnetic field effect; magnetoreception; optically detected EPR; EPR; CIDNP; CIDEP.

\section{INTRODUCTION}

Magnetic fields can alter the rate, yield, or product distribution of chemical reactions [1,2]. A wide variety of magnetic field effects (MFEs) are now known; they occur in diverse chemical systems at field strengths ranging from several Tesla, as at the heart of a superconducting magnet [3], down to only $\sim 40 \mu \mathrm{T}$, which is comparable to the field strength of the Earth [4]. In the latter case, this exquisite magnetic sensitivity arises even though the interaction between such a weak magnetic field and the molecules involved has an energy much smaller than the average thermal energy $k_{\mathrm{B}} T$. As we shall see, these phenomena arise through a detailed interplay of diffusion, reaction and quantum mechanical effects on the electron, and nuclear spins of the species involved. Measurement and analysis of MFEs has given rise to information on the reactivity, structure, and motion of these species. Such investigations are central to the field of endeavor known as "spin chemistry". This review gives a short introduction to spin chemistry, in a form accessible to graduate students and scientists new to the field. It summarizes the historical development of spin chemistry, sets out the essential theoretical framework for the analysis of MFEs, and presents representative examples of recent developments together with an assessment of the opportunities and challenges that lie ahead.

\section{HISTORICAL DEVELOPMENT}

Spin chemistry is an area of research centered around the influence of electron and nuclear spins on chemical reactivity. It encompasses research in chemically induced polarization effects in nuclear mag-

\footnotetext{
*Pure Appl. Chem. 81, 1-84 (2009). A collection of invited, peer-reviewed articles by the winners of the 2008 IUPAC Prize for Young Chemists.

¥E-mail: pubs-c@ rodgers.org.uk
} 
netic resonance (NMR) spectroscopy ("CIDNP") and in electron paramagnetic resonance (EPR) spectroscopy ("CIDEP"), in magnetic isotope effects, and in chemical MFEs, which are the focus here.

Apart from a few scattered reports, which were often irreproducible or lacked a theoretical interpretation, the first convincing chemical MFEs were observed in the late 1960s by Johnson for the triplet-exciton annihilation luminescence of anthracene crystals $[5,6]$. These experimental results were interpreted by Merrifield using a spin Hamiltonian approach [7].

Similar ideas emerged in the context of radiolysis, where the impact of high-energy particles creates radical ions which subsequently react with one another. Brocklehurst noted that electron spin is conserved during chemical reactions. Furthermore, in the hydrocarbon glasses used as a medium for radiolysis experiments, he estimated that electron spin relaxation would be relatively slow. Taking these ideas together, he predicted that the yield of recombination reactions between radical ions produced by radiolysis would show MFEs [8-10].

Also in the 1960s, several workers observed anomalous line shapes and enhanced signal intensities in the EPR spectra of radical reaction intermediates and later in the NMR spectra of the products of radical reactions [11]. These effects were dubbed "chemically induced dynamic electron (or nuclear) polarization" (CIDEP or CIDNP, respectively). They were interpreted independently by Kaptein and Oosterhoff [12] and by Closs [13] in terms of the radical pair mechanism (RPM) described below. The RPM was soon applied to the interpretation of chemical MFEs by Lawler and Evans [14]. This introduction of the RPM in the late 1960s marks the birth of spin chemistry.

The field grew rapidly in the following years. In the early 1970s, the triplet mechanism (TM) was introduced to explain entirely emissive CIDEP signals by Wan in Canada [15] and by Atkins and McLauchlan in Oxford [16]. Soon, Pedersen and Freed published a series of papers in which they developed a detailed theory for CIDEP [17-22]. A similarly detailed theory of chemical MFEs via the RPM arose a few years later with particular contributions from Brocklehurst $[9,10]$ and Schulten $[23,24]$. By the late 1970s, spin chemistry was burgeoning with input from research groups in Japan, Russia, Western Europe, and the United States.

Today, spin chemistry is a well-established branch of physical chemistry as illustrated by the citation analysis in Fig. 1. The methods of CIDNP and CIDEP have been developed and applied widely $[25,26]$. Chemical MFEs have formed the basis of a variety of reaction yield detected magnetic resonance (RYDMR) techniques [27]. These have been applied to study reactions in solids, liquids, and in heterogeneous media such as micelles. It is not possible to review such a broad range of developments in this short introductory article. Further details may be found in these reviews [1,28-32] and books [2,33-35].
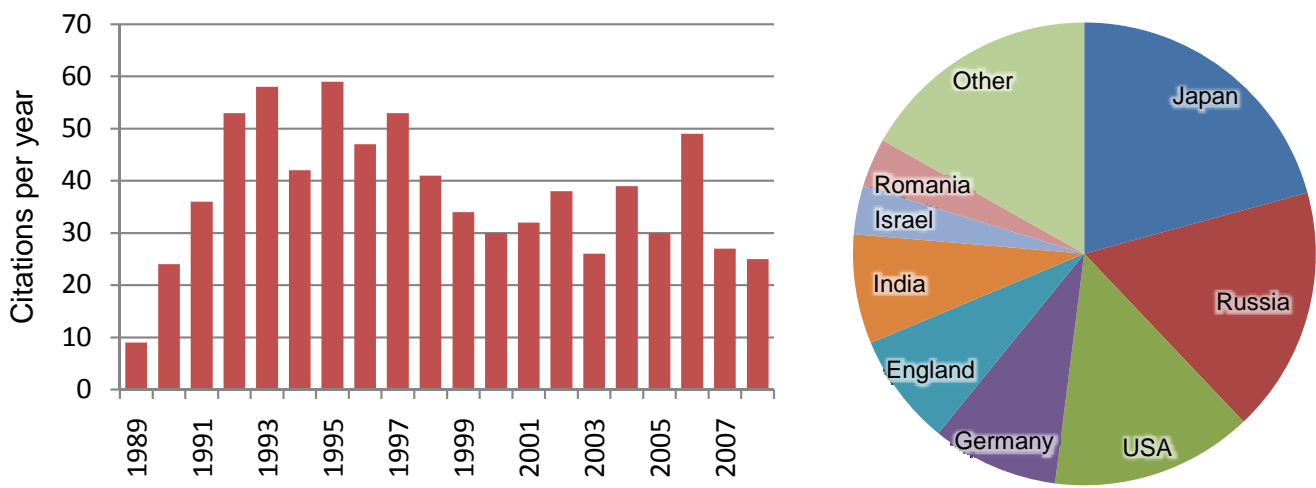

Fig. 1 Recent trends in spin chemistry research analyzed by year and by country, measured as citations of the landmark review of spin chemistry [1]. Data from ISI Web of Knowledge 2008. 


\section{THEORETICAL BACKGROUND}

Before turning our attention to recent developments in spin chemistry, it will be helpful to introduce the few elements of theory that are necessary to understand the work that follows. The following pages introduce the RPM, which provides the essential mechanistic framework for interpreting MFEs before reviewing the principal magnetic interactions that determine the nature of these effects.

\section{Radical pair mechanism}

The RPM [12,13,37] provides the mechanistic basis for the interpretation of most MFEs in chemical systems. The essential features, illustrated in Fig. 2, are as follows:

1. Ground-state precursor species "A B" are excited to produce two radicals whose electron spins are in a well-defined overall spin state, i.e., a spin-correlated radical pair (RP). For example, this excitation is often photochemical, where an incident photon is absorbed leading to the transfer of an electron from A to B, creating radical ions in a singlet state. Other possibilities include $\mathrm{H}$ atom transfer and thermolytic bond cleavage, which both produce uncharged RPs.

2. The singlet RPs undergo spin-selective reaction to produce the singlet product (SP) at a rate $k_{\mathrm{S}}$ or they react nonselectively to give a back-reaction product $\mathrm{X}$ at a rate $k_{\mathrm{S}}^{\mathrm{b}}$.

3. Coherent evolution of the RP spin state competes with these reactions, converting a proportion of the singlet (S) RPs back and forth into triplet (T) RPs. This $\mathrm{S} \leftrightarrow \mathrm{T}$ interconversion is driven by magnetic interactions in the RP and by any applied magnetic field.

4. The triplet RPs undergo their own spin-selective reaction to produce the triplet product TP at a rate $k_{\mathrm{T}}$ or again they react nonselectively to give the back-reaction product $\mathrm{X}$, this time at a rate $k_{\mathrm{T}}^{\mathrm{b}}$ which is not normally equal to $k_{\mathrm{S}}^{\mathrm{b}}$.

5. In many cases, the nonselective product $\mathrm{X}$ (or SP or TP) converts back into the precursor species "A B" which is then free again to begin step 1 .

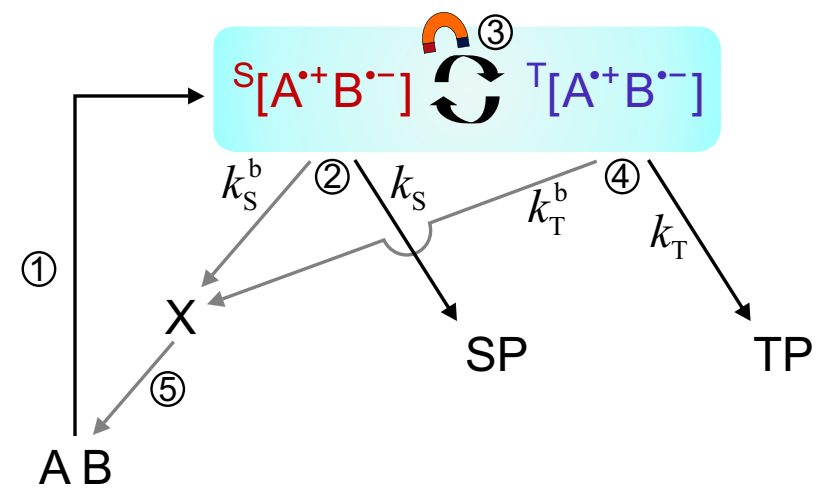

Fig. 2 Radical pair mechanism. After [36].

The key feature of the RPM is the spin evolution between singlet and triplet states in step 3 . Externally applied magnetic fields affect the rate and extent of this spin evolution and hence affect the yields of reaction products SP, TP, and X. Even weak magnetic fields whose interaction energies are far less than the thermal energy $k_{\mathrm{B}} T$ may cause significant changes in the yield of SPs. This does not contradict the laws of thermodynamics because generation of the RP in step 1 requires much more than the thermal energy $k_{\mathrm{B}} T$. The subsequent formation of products SP, TP, and $\mathrm{X}$ is determined by competitive kinetics. A helpful analogy is that of a railway train approaching a set of points. To drive the train, much energy is supplied by the locomotive just as in step 1, but the ultimate destination of the train will be 
determined by a small force used by the signal man to set the points. Even though the magnetic interactions with weak fields in step 3 are tiny, they may cause significant changes in the product yields.

\section{Spin dynamics}

Chemical MFEs arising from the RPM depend critically on the manner in which the overall RP spin state evolves. They derive from a detailed interplay of the interactions of the unpaired electron spins and of the kinetics of the radical reactions. We discuss first the electron spin evolution.

The unpaired electron spins and their selective reactivity are inherently quantum mechanical. In full generality, we ought to describe them by a wavefunction $\Psi\left(r_{i}, s_{i}, t\right)$ evolving under the influence of a Hamiltonian $\hat{H}(t)$, where $r_{i}$ and $s_{i}$ are the spatial and spin coordinates of the $i^{\text {th }}$ electron. Of course, the unpaired electrons would interact with all the other, paired electrons in each radical giving rise to electron correlation effects. Solving the Schrödinger equation for an RP described at this level of detail would be a formidable undertaking. Even after making the Born-Oppenheimer approximation, we would need to complete a full time-dependent quantum chemical calculation for every diffusive step. It is doubtful whether such a calculation would be feasible for any radicals of chemical interest.

Fortunately, it is not necessary to work in such generality. As in so much of science, the key to developing a useful theory of MFEs is to keep things as simple as possible, making as many simplifying assumptions as are permitted by experimental data. The first, and most essential, approximation that we make is to treat the time evolution of the electron spin and spatial coordinates separately. We do this by converting the full Hamiltonian into a spin Hamiltonian, which contains a number of empirical parameters, and the full wavefunction into a wavefunction containing only spin variables $[38,39]$. Since the spin Hamiltonian approach is ubiquitous in magnetic resonance, the form of the contributions arising from different magnetic interactions are known (see [40,41]). Furthermore, modern quantum chemical methods can calculate accurately many of the spin Hamiltonian parameters that we require.

\section{Vector operators}

Contributions to the RP spin Hamiltonian are most elegantly expressed using the notation of vector operators. For example, the bulk magnetization of a sample of electron spins is a quantum mechanical observable which may be given in terms of three separate expectation values: $S_{x}=\left\langle\hat{S}_{x}\right\rangle, S_{y}=\left\langle\hat{S}_{y}\right\rangle$, and $S_{z}=\left\langle\hat{S}_{z}\right\rangle$ that describe the components of the bulk magnetization along the $x$-, $y$-, and $z$-axes, respectively. The notation is simplified by defining an electron spin vector operator

$$
\hat{\mathbf{S}}=\hat{S}_{x} \mathbf{i}+\hat{S}_{y} \mathbf{j}+\hat{S}_{z} \mathbf{k}
$$

where $\mathbf{i}, \mathbf{j}$, and $\mathbf{k}$ are unit vectors along the $x$-, $y$-, and $z$-axes, respectively. The expectation value of this vector operator is the electron spin magnetization vector

$$
\langle\hat{\mathbf{S}}\rangle=\left\langle\hat{S}_{x}\right\rangle \mathbf{i}+\left\langle\hat{S}_{y}\right\rangle \mathbf{j}+\left\langle\hat{S}_{z}\right\rangle \mathbf{k}
$$

Vector operators provide a compact notation for the theory of RP reactions. For example, the singlet projection operator is

$$
\hat{P}^{\mathrm{S}}=\frac{1}{4}-\hat{\mathbf{S}}^{\mathrm{A}} \cdot \hat{\mathbf{S}}^{\mathrm{B}}
$$

where A and B label two electron spins and where the dot product "." between vector operators is interpreted as

$$
\hat{\mathbf{S}}^{\mathrm{A}} \cdot \hat{\mathbf{S}}^{\mathrm{B}}=\hat{S}_{x}^{\mathrm{A}} \hat{S}_{x}^{\mathrm{B}}+\hat{S}_{y}^{\mathrm{A}} \hat{S}_{y}^{\mathrm{B}}+\hat{S}_{z}^{\mathrm{A}} \hat{S}_{z}^{\mathrm{B}}
$$


This result is itself another operator.

Finally, for numerical calculations, one writes vector operators as Hilbert space matrices. For example, choosing the eigenvalues of $\hat{S}_{z}$ as basis functions, the electron spin vector operator is equal to

$$
\hat{\mathbf{S}}=\left(\begin{array}{cc}
0 & 1 / 2 \\
1 / 2 & 0
\end{array}\right) \mathbf{i}+\left(\begin{array}{cc}
0 & -i / 2 \\
i / 2 & 0
\end{array}\right) \mathbf{j}+\left(\begin{array}{cc}
1 / 2 & 0 \\
0 & -1 / 2
\end{array}\right) \mathbf{k}
$$

\section{Zeeman interaction}

The Zeeman interaction between the unpaired electron spins on each radical and the magnetic field obviously plays an essential role in the generation of an MFE. In a rotationally symmetric potential, such as in an atom, the Zeeman interaction between an electron and a magnetic field is [42]

$$
\hat{H}_{\mathrm{Z}}=\mu_{\mathrm{B}}\left(\hat{\mathbf{L}}+g_{\mathrm{e}} \hat{\mathbf{S}}\right) \cdot \boldsymbol{B}
$$

where $\mu_{\mathrm{B}}$ is the Bohr magneton, $\hat{\mathbf{L}}$ is the electron orbital angular momentum vector operator, $g_{\mathrm{e}}$ is the free electron $g$-factor, and $\boldsymbol{B}$ is the magnetic field vector.

In a molecule, the electric potential field is no longer rotationally symmetric. As a consequence, there are complicated interactions between the electrons, which are responsible for spin-orbit coupling. In molecules, the orbital angular momentum quantum number $\hat{\mathbf{L}}$ is no longer well defined, so we introduce instead the tensor " $g$ " to account for these complexities. For a particular molecule in a particular electronic state, the Zeeman interaction may therefore be written [42]

$$
\hat{H}_{\mathrm{Z}}=\mu_{\mathrm{B}} \hat{\mathbf{S}} \cdot g \cdot \boldsymbol{B}
$$

The g-tensor is often determined empirically from EPR spectra. Alternatively, modern density functional theory (DFT) methods allow reasonably accurate calculation of the g-tensor for molecules as large as a small protein [43].

Chemical MFEs are frequently studied in small organic molecules and weak fields, where it is reasonable to assume that the g-tensor is that of a free electron. Hence,

$$
\hat{H}_{\mathrm{Z}}=g_{\mathrm{e}} \mu_{\mathrm{B}} \hat{\mathbf{S}} \cdot \boldsymbol{B}=-\gamma_{\mathrm{e}} \hbar \hat{\mathbf{S}} \cdot \boldsymbol{B}
$$

in which $\gamma_{\mathrm{e}}=-g_{\mathrm{e}} \mu_{\mathrm{B}} / \hbar$ is the electron magnetogyric ratio. Writing this in angular frequency units as is common gives

$$
\hat{H}_{\mathrm{Z}}=-\gamma_{\mathrm{e}} \hat{\mathbf{S}} \cdot \boldsymbol{B}
$$

The nuclei in a RP also experience Zeeman interactions with a magnetic field, but these are negligible because nuclear magnetogyric ratios are many times smaller than that of the electron. This is particularly true for small applied fields where the nuclear Zeeman interaction is much weaker than the hyperfine interaction.

\section{Zeeman resonance}

If the applied magnetic field contains an oscillating (radiofrequency or microwave) component $\boldsymbol{B}_{1}$ at a frequency $v_{\mathrm{rf}}$ in addition to the static field $\boldsymbol{B}_{0}$, then a vast range of magnetic resonance experiments become possible. In RP reactions, these effects often obey the same selection rules as in high-field EPR. There is normally a strong "Zeeman resonance" when the oscillating field is in resonance with the energy level splitting caused by the static field. More specifically, if we define the $z$-axis to lie along the 
magnetic field orientation so that $\boldsymbol{B}=\boldsymbol{B}_{0} \mathbf{k}$, then to first order, the Zeeman energy for an electron spin eigenstate $\hat{S}_{z}\left|m_{s}\right\rangle=m_{s} \hbar\left|m_{s}\right\rangle$ is

$$
E_{\mathrm{Z}}=-\gamma_{\mathrm{e}} \hbar m_{s} B_{0}
$$

The selection rule for transitions between these states is $\Delta m_{s}= \pm 1$. This transition is, therefore, in resonance with radiofrequency (RF) (or microwave) photons of frequency $v_{\mathrm{rf}}$ where

$$
h v_{\mathrm{rf}}=\Delta E_{\mathrm{Z}}=\left|\gamma_{\mathrm{e}}\right| \hbar B_{0}
$$

and hence a Zeeman resonance might be expected for frequencies

$$
v_{\mathrm{rf}}=\frac{\left|\gamma_{\mathrm{e}}\right|}{2 \pi} B_{0}=\left(28.025 \mathrm{MHz} \mathrm{mT}^{-1}\right) \times B_{0}
$$

or equivalently at field strengths

$$
B_{0}=\frac{2 \pi}{\left|\gamma_{\mathrm{e}}\right|} v_{\mathrm{rf}}=\left(35.682 \mu \mathrm{T} \mathrm{MHz}{ }^{-1}\right) \times v_{\mathrm{rf}}
$$

as will be seen below.

\section{Hyperfine interaction}

The Zeeman interaction couples the unpaired electron spin of a radical with an external applied magnetic field. In contrast, the hyperfine interaction couples the unpaired electron spin with the internal magnetic field from the spins of magnetic $I>0$ nuclei in the radical. The hyperfine interaction is made up of two contributions [41].

The first of these is the direct dipolar interaction between the magnetic moments of the unpaired electron and the nucleus in question. When MFEs are measured in isotropic liquids, this dipolar contribution is averaged to zero by rapid tumbling of the radicals. In the solid state or in oriented media such as a liquid crystal, they make the hyperfine interaction anisotropic, i.e., it depends not only on the relative orientation of electron and nuclear spins but also on the orientation of these spins with respect to the molecular frame of the radicals.

The other contribution to the hyperfine interaction is known as the Fermi contact interaction. It is caused by the magnetic interaction of the electron and nuclear spins when the electron is within the nucleus. As such, it is proportional to the s-orbital character of the singly occupied molecular orbital (SOMO) around the nucleus in question. The Fermi contact interaction is isotropic; it does not depend on the orientation of the electron or nuclear spins with respect to the molecule, but only on their relative orientation. The Fermi contact interaction is described by

$$
\hat{H}_{\mathrm{HFI}}=a_{N i} \hat{\mathbf{S}}^{N} \cdot \hat{\mathbf{I}}^{N i}
$$

where $a_{N i}$ is the isotropic hyperfine coupling (HFC) constant for interaction between an electron $\hat{\mathbf{S}}^{N}$ and nuclear spin $\hat{\mathbf{I}}^{N i}$.

Overall, the hyperfine interaction is described by

$$
\hat{H}_{\mathrm{HFI}}=\hat{\mathbf{S}}^{N} \cdot \mathbf{A}_{N i} \cdot \hat{\mathbf{I}}^{N i}
$$


where $\mathbf{A}_{N i}$ is the hyperfine tensor for radical $N$ and nucleus $i$ and $a_{N i}=\operatorname{trace}\left(\mathbf{A}_{N i}\right) / 3$. The hyperfine interaction is proportional to the magnetogyric ratio $\gamma_{N}$ of the nucleus concerned. For example, replacing hydrogen by deuterium will reduce the hyperfine interaction by approximately $\gamma_{\mathrm{H}} / \gamma_{\mathrm{D}} \approx 6.5$ times.

The hyperfine interaction may be calculated accurately using DFT $[44,45]$ from the electron density in the SOMO. The anisotropic part is determined by the broad spatial distribution of the unpaired electron, whilst the isotropic part is determined by the unpaired electron density at the nucleus.

\section{Exchange interaction}

One normally thinks of the radicals in an RP as separate entities. Within each radical, Coulombic forces between the electrons and the nuclei determine the radical's geometry and electronic structure. These, in turn, produce contributions to the radical spin Hamiltonian characterized by the g-tensor and hyperfine tensors.

However, this picture is incomplete. For a full treatment, one ought also to include the Coulombic interactions between all the nuclei and electrons on one radical with those on the other radical. Figure 3 illustrates these effects for an RP comprising two hydrogen atoms $\mathrm{H}^{\bullet}$, i.e., for a $\mathrm{H}_{2}$ molecule that has been stretched or compressed.

- As the hydrogen atoms are brought together, their electronic wavefunctions begin to overlap. The interaction between the atomic wavefunctions produces bonding $1 \sigma_{\mathrm{g}}$ and anti-bonding $2 \sigma_{\mathrm{u}}{ }^{*}$ molecular orbitals (MOs) (one-electron molecular wavefunctions) as shown in Fig. 3A.

- $\quad$ According to the orbital approximation, the $n$-electron wavefunction for the RP as a whole is given as a symmetrized product of these one-electron MOs.

- The lowest energy state ( $n$-electron wavefunction) arises when both electrons occupy the $1 \sigma_{\mathrm{g}}$ MO. According to the Pauli principle, this is only possible when the electron spins are paired to give a ${ }^{1} \Sigma_{\mathrm{g}}{ }^{+}$state.

- The next state arises when one electron is promoted to the $2 \sigma_{\mathrm{u}}{ }^{*} \mathrm{MO}$. This time, the Pauli principle permits ${ }^{3} \Sigma_{\mathrm{u}}{ }^{+}$and ${ }^{1} \Sigma_{\mathrm{u}}{ }^{+}$states.

- According to Hund's rules, the ${ }^{3} \Sigma_{\mathrm{u}}{ }^{+}$state is the lower in energy.

- Figure 3B shows the energies of the ${ }^{1} \Sigma_{\mathrm{g}}{ }^{+}$ground state (labeled "S" for singlet) and the ${ }^{3} \Sigma_{\mathrm{u}}{ }^{+}$first excited state (labeled " $\mathrm{T}$ " for triplet). The energy difference between these states, denoted $J(r)$, depends on the radical separation $r$.
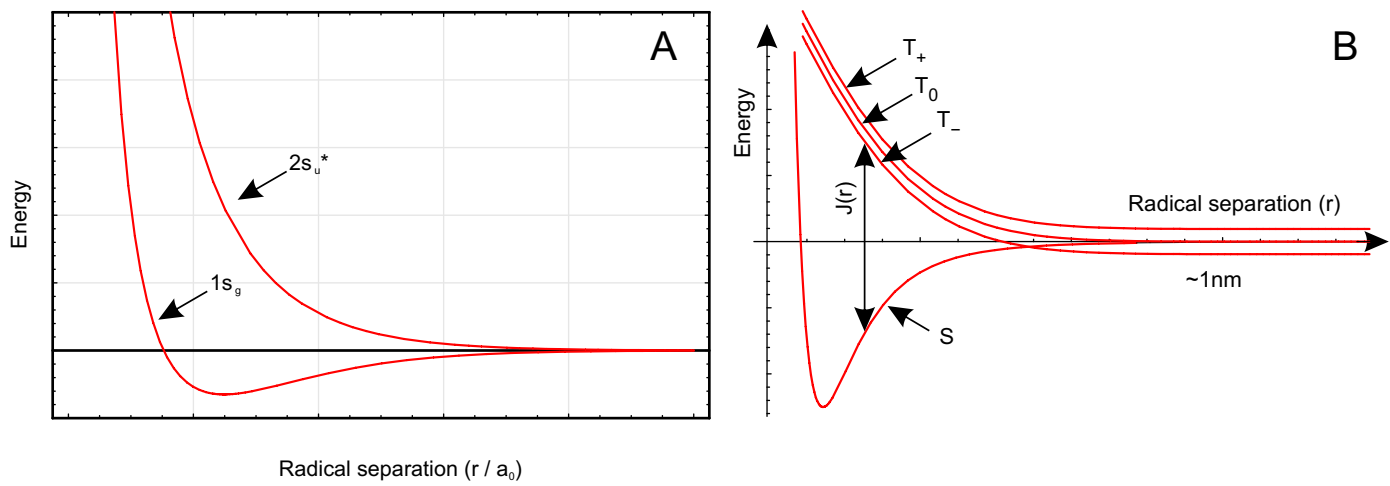

Fig. 3 Exchange interaction in RPs. (A) First two LCAO MO energies for the hydrogen molecular ion $\mathrm{H}_{2}{ }^{+}$as a function of inter-nuclear separation. (B) Approximate energies for the ground ( ${ }^{1} \Sigma_{\mathrm{g}}^{+}$"S") and first excited ( ${ }^{3} \Sigma_{\mathrm{u}}^{+}$" $\mathrm{T}$ ") states of a pair of hydrogen atoms as a function of inter-nuclear separation $r$. When $r$ is short, the hydrogen atoms are bonded and should best be thought of as a $\mathrm{H}_{2}$ molecule. At long separations, we could better describe this system as a $\left[\mathrm{H}^{\bullet} \mathrm{H}^{*}\right] \mathrm{RP}$. The separation between $\mathrm{S}$ and $\mathrm{T}_{0}$ states is labeled $J(r)$. The $\mathrm{T}_{ \pm}$and $\mathrm{T}_{0}$ states are split apart due to the Zeeman interaction. 
To describe the spin evolution of an RP where the radicals approach closely enough for inter-radical electron correlation and bonding effects to set in, we use the Heisenberg-Dirac-van Vleck (HDVV) spin Hamiltonian [46]

$$
H_{\mathrm{ex}}=-J \hat{\mathbf{S}}^{\mathrm{A}} \cdot \hat{\mathbf{S}}^{\mathrm{B}}
$$

The empirical parameter $J$ is chosen so that the HDVV eigenstates have the same energy separation as the ground and first excited states when the Schrödinger equation is solved for the "molecule" comprising both radicals taken together. For an RP, this means that $J$ is the difference in energy between the lowest energy singlet and triplet states.

In spin chemistry, the exchange interaction is normally assumed to decrease exponentially with increasing RP separation and to be independent of the orientation of the radicals and of the solvent. In other words

$$
J(r) \approx J_{0} e^{-r / r_{J}}
$$

where $J_{0}$ is the magnitude, $r$ is the inter-radical separation and $r_{J}$ is a range parameter. This relationship has extensive empirical support in proteins when $r$ is taken to be the edge-to-edge distance between electron transfer centers [47]. Illustrative values are $J_{0} \sim 2 \times 10^{17} \mathrm{rad} \mathrm{s}^{-1}$ and $r_{J} \sim 50 \mathrm{pm}$ [37].

However, the values of the parameters vary considerably with the nature of the radicals and the intervening medium. Hence, it is a formidable task to calculate $J$ for radicals in solution. Quantum chemically, this is equivalent to calculating the energy levels in a loosely bound "molecule" comprising the radicals A and B together with any solvent molecules in proximity. The solvent molecules can be particularly significant because they can mediate the interaction between the unpaired electrons on each radical in a process known as superexchange. A few attempts have been made to perform such calculations [46] and to compare experimental and theoretical results in solid-state lophyl RPs [48] and in linked donor-acceptor diad compounds [49]. A full understanding of the RP exchange interaction is still awaited.

In solution-phase RP reactions, exchange acts to prevent singlet-triplet interconversion. Hence, whenever the radicals are close enough that exchange dominates the RP spin Hamiltonian, no MFEs may arise. For example, exchange suspends RP spin evolution during diffusive encounters as discussed later.

\section{Dipolar interaction}

Each unpaired electron spin also experiences a magnetic field from the other unpaired electron. These dipolar interactions may be described by the Hamiltonian [50]

$$
\hat{H}_{\mathrm{D}}=\frac{\mu_{0} \mu_{\mathrm{B}}^{2} g_{\mathrm{A}} g_{\mathrm{B}}}{4 \pi \hbar^{2} r^{3}}\left[\hat{\mathbf{S}}^{\mathrm{A}} \cdot \hat{\mathbf{S}}^{\mathrm{B}}-\frac{3}{r^{2}}\left(\hat{\mathbf{S}}^{\mathrm{A}} \cdot \mathbf{r}\right)\left(\hat{\mathbf{S}}^{\mathrm{B}} \cdot \mathbf{r}\right)\right]
$$

in angular frequency units where $g_{N}$ are the electron g-factors for each radical and $\hat{\mathbf{S}}$ are the electron spin vector operators. Equation 18 is, strictly speaking, only valid for point dipoles separated by a vector $\mathbf{r}$. In real molecules, $\mathbf{r}$ is closely approximated by the vector between the "centers" of the SOMOs on each radical. Simple algebraic manipulations allow eq. 18 to be written

$$
\hat{H}_{D}=\hat{\mathbf{S}}^{\mathrm{A}} \cdot D \cdot \hat{\mathbf{S}}^{\mathrm{B}}
$$

in terms of the dipolar tensor $D$. At high field, diffusive motion of one radical around the other causes the dipolar interaction to average to zero. In weak fields, the situation is more complicated. Detailed 
calculations show that at weak fields, the dipolar interaction acts generally to suppress MFEs [51] unless the exchange and dipolar interactions are of similar energies, in which case MFEs are still predicted to arise [52].

\section{Field strength regimes}

In fields $B_{0}<50 \mathrm{mT}$, the singlet-triplet interconversion responsible for MFEs is governed primarily by the isotropic electron Zeeman and the hyperfine interactions. This is known as the hyperfine mechanism. At higher fields, the situation is rather different. There, singlet-triplet interconversion arises for the most part because of small differences in the g-tensors of the two radicals, in what is known as the $\Delta g$-mechanism. Figure 4 summarizes this change in mechanism.

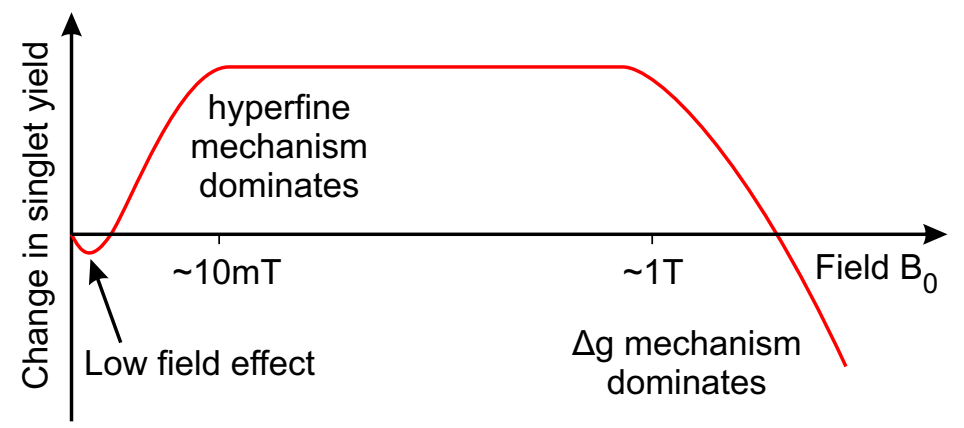

Fig. 4 Typical MFEs in organic RPs from zero field up to a few Tesla.

\section{APPLICATIONS IN PHYSICAL CHEMISTRY}

Spin chemistry has made contributions in diverse areas of science: in chemistry, physics, biology, and materials science, to name but a few. The RPM presented in Fig. 2 is realized in a wealth of different systems. In each case, the key features of spin-selective competitive kinetics remain as in Fig. 2, but the details of the physical model change. The following pages survey some applications of the RPM in liquids and in the solid state. To give a flavor of the field, recent developments in three broad areas are covered below. The final section of this review is a personal perspective on one of the most interesting challenges that lies ahead in spin chemistry.

\section{Reaction-diffusion in liquids}

Studies of chemical MFEs have provided a good deal of insight into the chemical physics of liquids and solutions. Radical pair mechanisms, outlined previously in Fig. 2, revolve around kinetic competition that determines which products are formed from RP intermediates. In the general scheme above, this competition was governed by first-order rate constants $k_{\mathrm{S}}$, $k_{\mathrm{S}}^{\mathrm{b}}$, etc. In the liquid phase, the magnitude and features of CIDNP, CIDEP, and chemical MFEs depend critically on the diffusive motion of the RP involved. The kinetics controlling the reaction of the RPs may be understood more explicitly in terms of the reaction-diffusion form of the RPM shown in Fig. 5, the key features of which are as follows:

- The precursor(s) react to form an RP in a well-defined spin state, e.g., the singlet ${ }^{\mathrm{S}}\left[\mathrm{A}^{\bullet} \mathrm{B}^{\bullet}\right]$. The $\mathrm{RP}$ is excited by much more than $k_{\mathrm{B}} T$, which ensures that its subsequent fate is controlled kinetically and not by equilibrium thermodynamics.

- $\quad$ For $\sim 500$ ps after this, the radicals are caught in very close proximity by a "cage" of surrounding solvent molecules. They collide frequently with one another during this first encounter. A proportion of the RPs react during these frequent collisions to form the SP. This step, known as "pri- 
mary geminate recombination", is insensitive to magnetic fields [53] because the exchange interaction is strong at short separation which inhibits $\mathrm{S} \leftrightarrow \mathrm{T}$ interconversion. Experimentally, it is often suppressed using modulation and phase-sensitive detection techniques such that it may, to all intents and purposes, be ignored.

- $\quad$ Some RPs, however, do not react in these first instants, and separate instead, making a "diffusive excursion" in the surrounding liquid. The exchange interaction between electron spins that was very large when the radicals were in close proximity is now much less significant. Hence, the RP is free to undergo $\mathrm{S} \leftrightarrow \mathrm{T}$ interconversion under the influence of molecular hyperfine interactions and, crucially, under the influence of the applied magnetic field.

- At the end of a diffusive excursion, some RPs separate for good, whence they react ultimately, e.g., by $\mathrm{H}$ atom abstraction from the solvent, to form the "escape product" (EP).

- $\quad$ Other RPs re-encounter one-another, whereupon the radicals are once more held within a solvent cage and undergo frequent collisions, which afford them opportunity to react with one another. Such reactions are known as "secondary geminate recombination".

- Whether the radicals react during a re-encounter depends on the spin evolution during the preceding diffusive excursion. For example, in the reaction between pyrene (Py) and $N, N$-dimethylaniline (DMA) shown in Fig. 6, spin is conserved during the back-electron transfer reaction to form an exciplex. This means that the radicals may only undergo back-electron transfer during a re-encounter when the RP is in an overall singlet spin state. We refer to such products as the "singlet product" (SP). RPs in a triplet state can do nothing other than to separate once more.

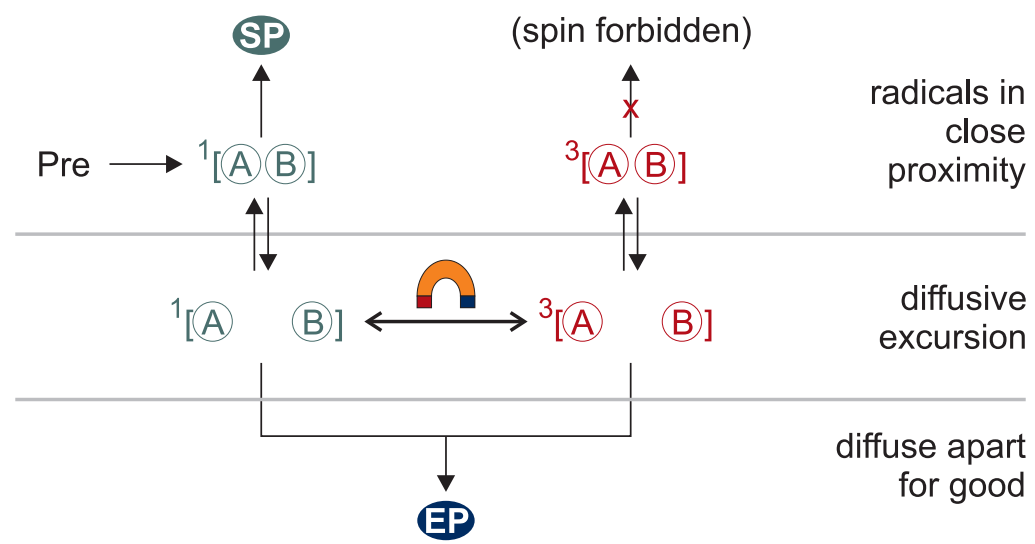

Fig. 5 Reaction-diffusion RPM in liquids. 


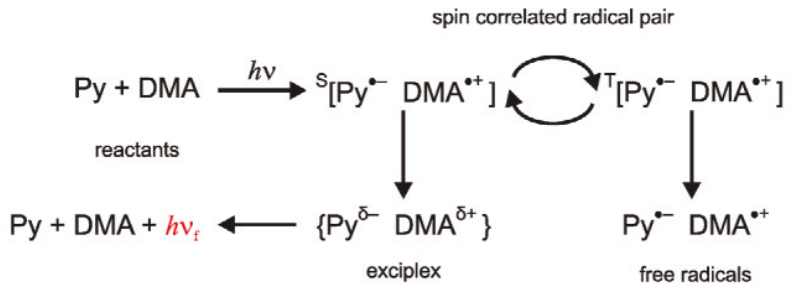

(a) Essential steps in the photoinduced reaction of pyrene (Py) and $N, N$-dimethylaniline (DMA) via RP state $\left[\mathrm{Py}^{\circ-} \mathrm{DMA}^{*+}\right]$ which is responsible for the magnetic field-sensitivity of the exciplex fluorescence. $h v$ signifies the incoming $\mathrm{UV}$ radiation and $h v_{\mathrm{f}}$ the observed exciplex fluorescence.

Py:

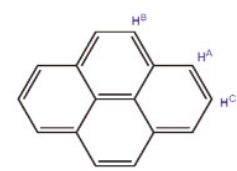

DMA:

(b) Structures of pyrene (Py) and DMA

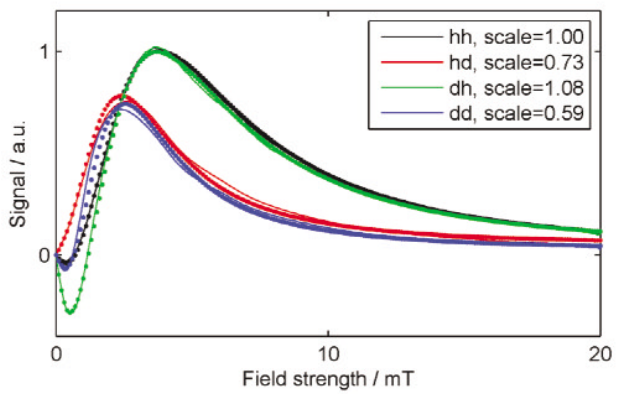

(c) Best fits to the experimental data using Tikhonov regularization applied to all four isotopomer data sets simultaneously in order to produce a single re-encounter probability $f(t)$. The experimental data were multiplied by optimized values of "scale" before using Tikhonov regularization. Notice that the fit is exemplary, except for small deviations in the dd isotopomer curve. This figure complements curve $\mathrm{C}$ in subfigure (d).
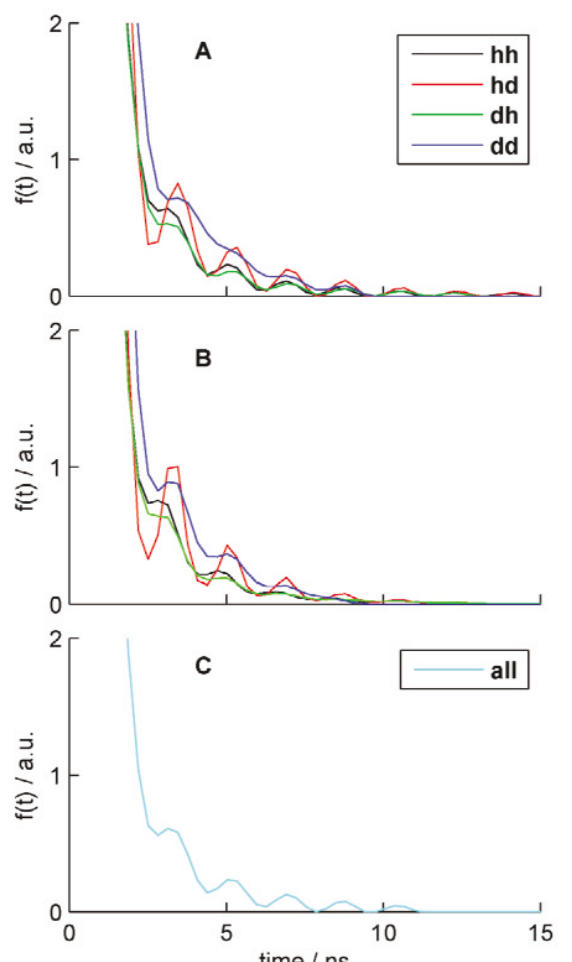

(d) Best recovered $f(t)$ for: (A) Tikhonov regularization; and (b) maximum entropy regularization for each of the isotopomer combinations. (C) Tikhonov regularization recover of a single $f(t)$ to fit all four isotopomer combinations simultaneously.

Fig. 6 Regularization methods allow details of the diffusive motion of RPs to be recovered from experimental MFE data. Adapted from [53].

\section{Solution-phase experiments}

A number of different approaches have been taken to measuring chemical MFEs in the liquid phase. Some of these approaches are presented here, serving as both an introduction to experimental work in the field and an illustration of the reaction-diffusion theory presented above. A multitude of names have been given to these closely related experiments. 
In these experiments, the RP spins typically evolve under a Hamiltonian

$$
\hat{H}=\sum_{N=\{A, B\}}\left\{-\gamma_{\mathrm{e}} \boldsymbol{B}_{0} \cdot \hat{\mathbf{S}}^{N}-\gamma_{\mathrm{e}} \boldsymbol{B}_{1}(t) \cdot \hat{\mathbf{S}}^{N}+\sum_{i} a_{N i} \hat{\mathbf{S}}^{N} \cdot \hat{\mathbf{I}}^{N i}\right\}-e^{-r / r_{J}} J_{0} \hat{\mathbf{S}}^{\mathrm{A}} \cdot \hat{\mathbf{S}}^{\mathrm{B}}
$$

using the notation defined above with $\boldsymbol{B}_{0}$ denoting the static (time-independent) magnetic field and $\boldsymbol{B}_{0}(t)$ its time-dependent component. Since a typical RP lifetime is of the order of $10 \mathrm{~ns}-1 \mu \mathrm{s}$, fields with frequency $v_{\mathrm{rf}}>1 \mathrm{MHz}$ could show resonant effects. It transpires that in weak static fields, radiofrequencies $(\mathrm{MHz})$ are most appropriate, whilst microwave frequencies $(\mathrm{GHz})$ are best in strong $(>1 \mathrm{~T})$ static fields. Experimental measurements of chemical MFEs customarily record the singlet or triplet product yield as a function of the field strengths $B_{0}$ and $B_{1}$, or as a function of the frequency of the timedependent field.

Measuring the product yield as a function of the static field strength $B_{0}$, without RF, generates what is known as a MARY (magnetic field modulation of the reaction yields) "spectrum" [54]. Examples and references to the literature are given below in connection with Figs. 6 and 7.

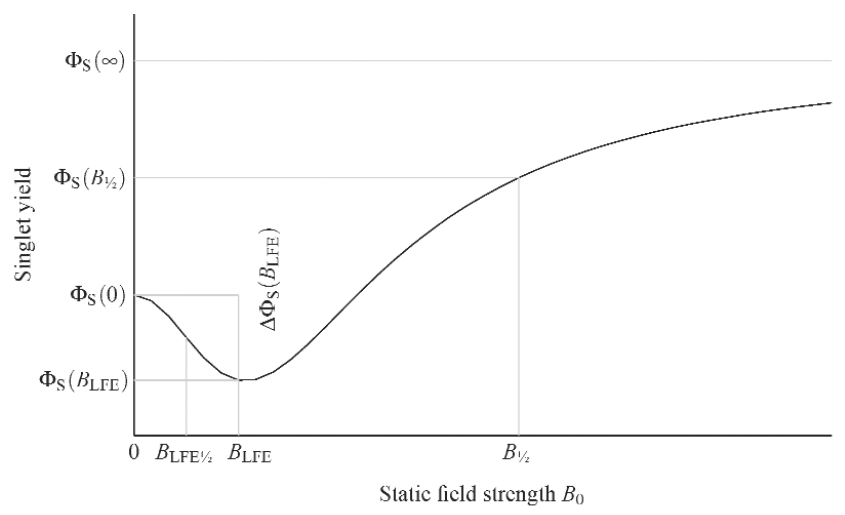

(a) MARY curve for a one-proton radical pair marked with the principal empirical parameters discussed in the main text.

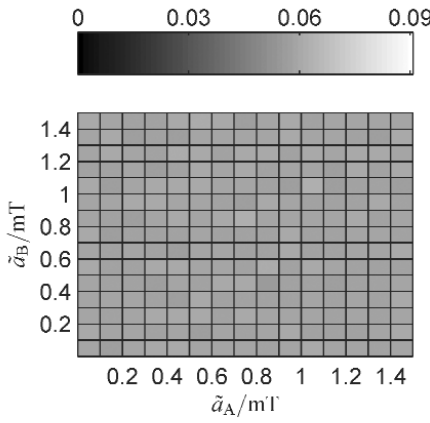

(b) Mean LFE depth $\Delta \Phi_{\mathrm{S}}\left(B_{\mathrm{LFE}}\right)$ when $k=$ $0.01 \mathrm{~s}^{1}$. Values range between 0.05 and 0.06 .

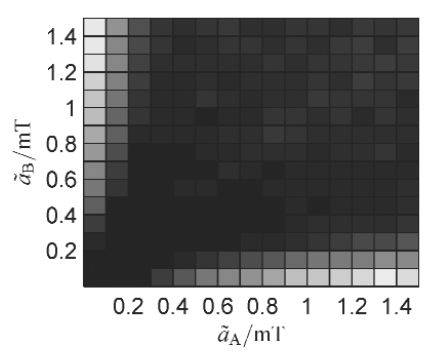

(c) Mean LFE depth $\Delta \Phi_{\mathrm{S}}\left(B_{\mathrm{LFE}}\right)$ when $k=$ $3 \times 10^{7} \mathrm{~s}^{1}$. Values range between 0.00 and 0.08 .

Fig. 7 Top: Characterizing MARY curves. Bottom: LFE depth $\Delta \Phi_{\mathrm{S}}\left(B_{\mathrm{LFE}}\right)$ determined by Monte Carlo calculations in an ensemble of 12737 RPs. The color of each rectangle summarizes the results from those members of the ensemble having effective HFCs that fall within it. Rate constants $k$ were selected to be less than and comparable to the effective HFCs. 
An OMFE (oscillating magnetic field effect) spectrum is acquired by measuring the product yield whilst applying only an RF field, whose strength is fixed and whose frequency is swept, e.g., from 5 to $80 \mathrm{MHz}$ to elucidate resonant responses of the RP spin system [55-57].

When static and time-dependent fields are applied simultaneously, it is possible to observe strong field effects caused by resonances with the Zeeman energy splitting in the RP. When applied in weak $(<100 \mathrm{mT})$ static fields, with RF oscillating fields and when the reaction yield is monitored optically, these experiments are known as optically detected electron paramagnetic resonance (OD-EPR) [58-63]. Examples are given below in connection with Figs. 8 and 9.

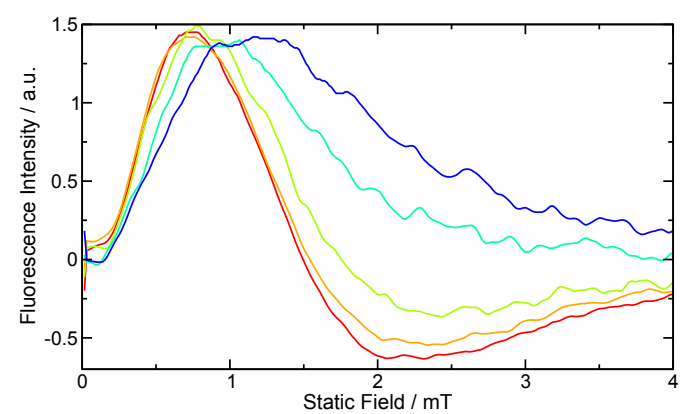

(a) $5 \mathrm{MHz}$ experimental data

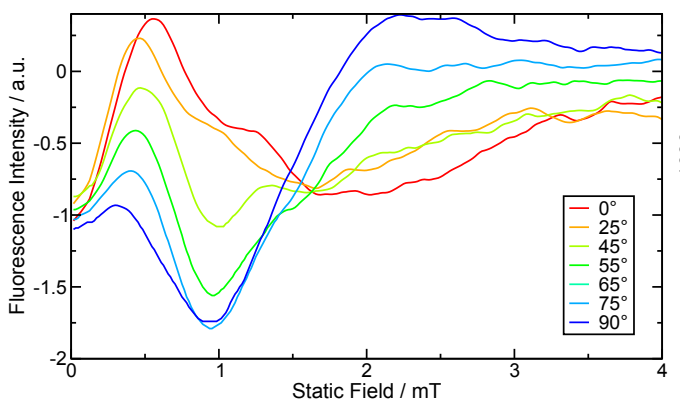

(c) $20 \mathrm{MHz}$ experimental data

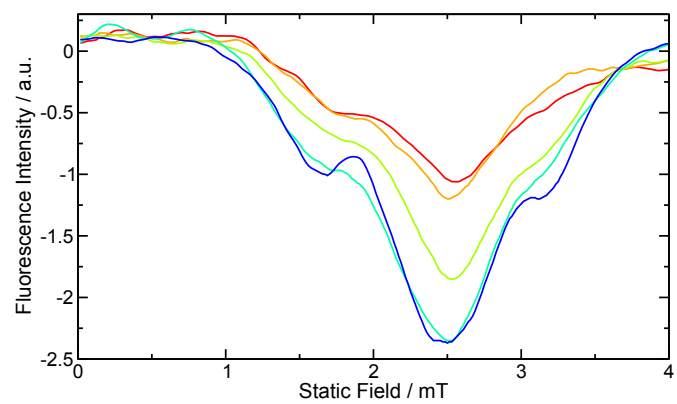

(e) $65 \mathrm{MHz}$ experimental data

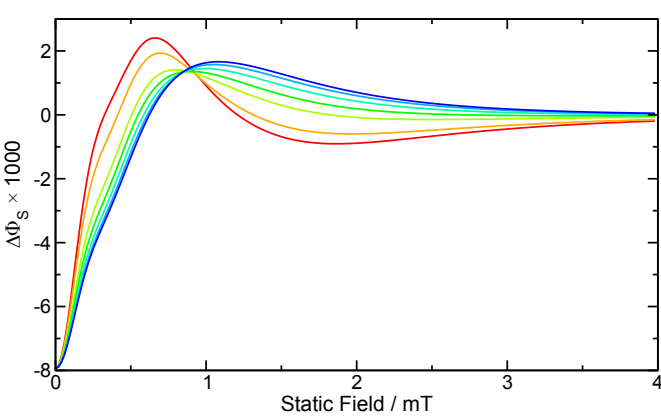

(b) $5 \mathrm{MHz}$ simulation

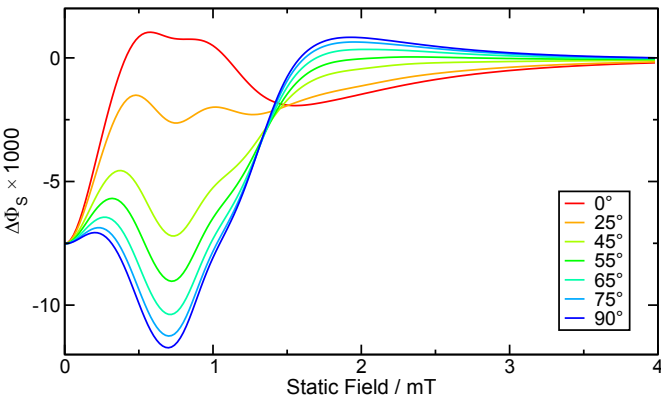

(d) $20 \mathrm{MHz}$ simulation

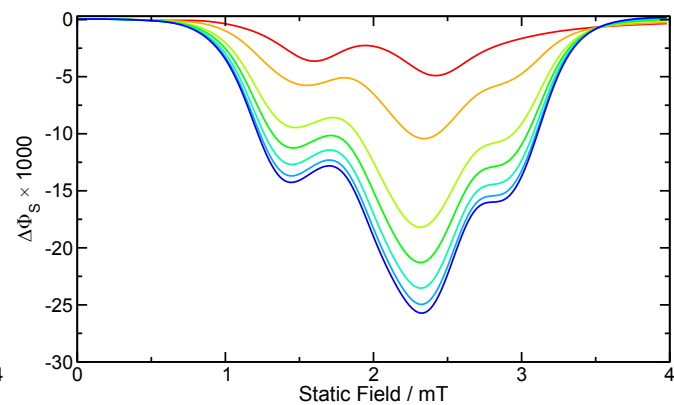

(f) $65 \mathrm{MHz}$ simulation

Fig. 8 Experimental (left) and simulated (right) low-field optically detected EPR spectra of [Py-d $\left.{ }_{10}^{++} 1,3-\mathrm{DCB}^{\circ-}\right]$ at RFs of $v_{\mathrm{rf}}=5 \mathrm{MHz}$ (top), $20 \mathrm{MHz}$ (center) and $65 \mathrm{MHz}$ (bottom). Values of $\theta$ are as indicated in the legend. The same colors are used for all plots, even though experimental data were not collected for some combinations of $v_{\mathrm{rf}}$ and $\theta$. The simulations were performed using $B_{1}=0.3 \mathrm{mT}$ and $k=4 \times 10^{7} \mathrm{~s}^{-1}$. The experimental spectra measure changes produced by the RF field in the exciplex fluorescence of the singlet RP. 

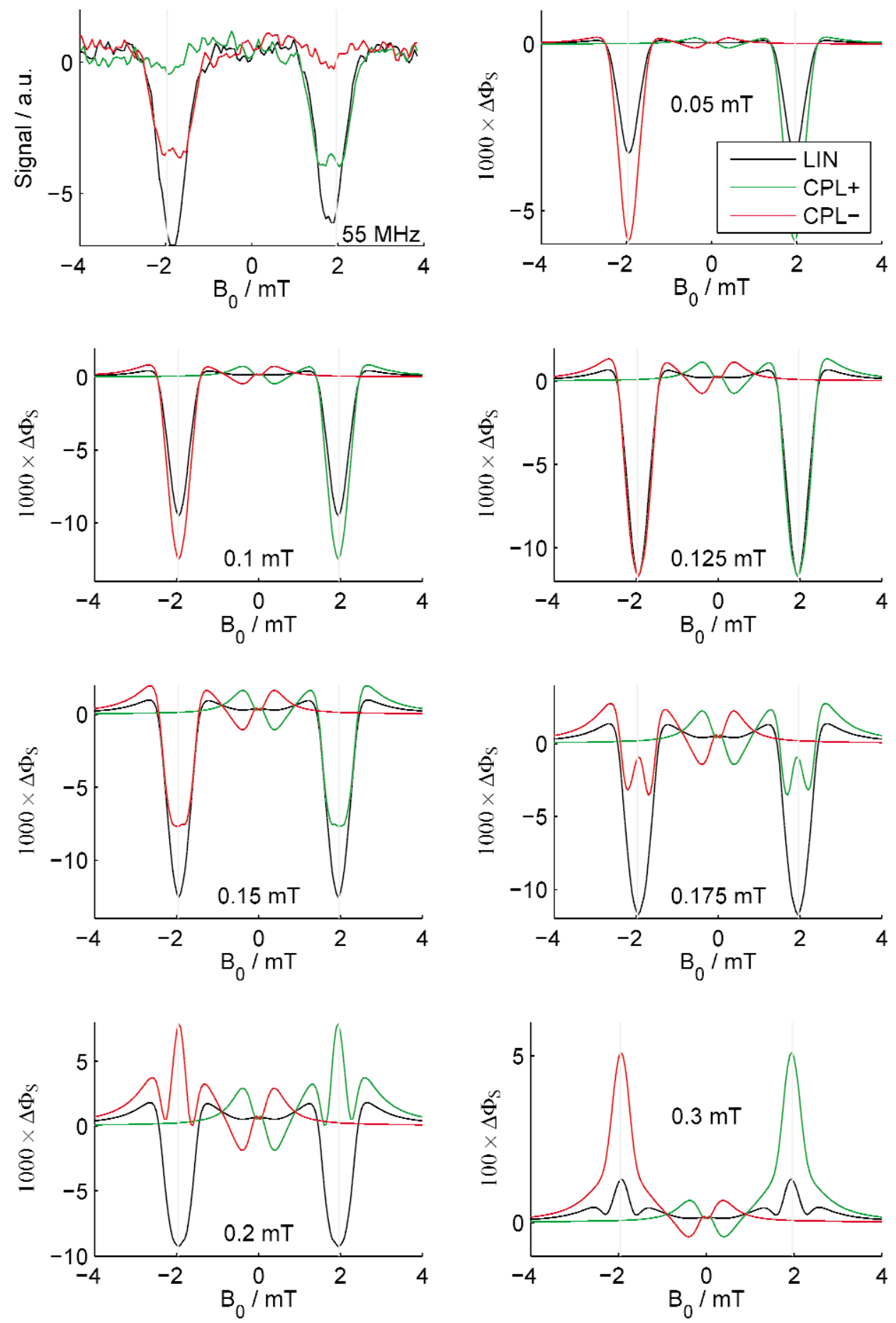

Fig. 9 RP reactions can show markedly different field effects depending on the polarization of the applied RF field. Top left: Experimental MARY- $v$ spectra measured in the $\left[\mathrm{Py}-\mathrm{d}_{10}^{\bullet+} 1,4-\mathrm{DCB}^{\bullet-}\right] \mathrm{RP}$ at $v_{\mathrm{rf}}=55 \mathrm{MHz}$. Other figures: Simulations made with $\gamma$-COMPUTE or the rotating frame transformation. Parameters: RMS RF field strength $B_{1}$ is marked on each figure, HFCs of $4 \times 0.083 \mathrm{mT}\left({ }^{2} \mathrm{D}\right)$ on $\mathrm{Py}-\mathrm{d}_{10}^{\bullet+}$; and $2 \times 0.181 \mathrm{mT}\left({ }^{14} \mathrm{~N}\right)$ on $1,4-\mathrm{DCB}^{\bullet-}, k=3 \times$ $10^{7} \mathrm{~s}^{-1}, n=64, \theta=\pi / 2$ and $v_{\mathrm{rf}}=55 \mathrm{MHz}$. Gray vertical lines show the Zeeman resonance static field. 
With stronger fields ( $>100 \mathrm{mT}$ ), the Zeeman resonance occurs at microwave frequencies, which necessitates using apparatus that strongly resembles a traditional EPR spectrometer; the experiment is then known as RYDMR [27].

Theoretically, weak magnetic fields acting via the RPM may produce changes in product yield up to $40 \%$ in simple model systems [64]. In practice, however, the effects observed are typically $\sim 1 \%$. Reliable detection of these small effects is most easily achieved in systems where the RPM is cyclic (the gray pathways in Fig. 2) by modulating the applied magnetic field and employing phase-sensitive detection. Continuous excitation of RPs typically allows one to ignore the photochemistry leading to generation of the RP itself, which simplifies the analysis considerably.

On the other hand, it is also possible to generate the RPs with a pulsed excitation either by flash photolysis with a laser source [4,65], or by pulse radiolysis using ionizing radiation such as X-rays [66]. MFEs on the ensuing RP reaction appear as changes in the lifetimes of the species involved, which may be monitored by fluorescence or by optical absorption. These experiments are applicable to a far wider range of reactions, because practically any species may be detected sensitively by optical absorption at an appropriate wavelength. They provide rich data on the kinetics of the reaction under investigation, although this can be more challenging to interpret theoretically compared to data from continuous RP generation.

Alternatively, the reaction may be following by monitoring the decay in electron spin polarization of the RP state in a time-resolved electron paramagnetic resonance (TR-EPR) experiment [67]. Finally, MFEs may be detected via enhanced nuclear polarizations in the diamagnetic reaction products measured in an NMR spectrometer moments after excitation and reaction in a variable magnetic field. The reaction of interest is run in an NMR tube in an appropriate weak magnetic field; the tube is then rapidly transferred into a high-field NMR spectrometer whereupon a spectrum is collected. The MFEs manifest as non-equilibrium polarizations of the nuclear spins in the diamagnetic reaction products, producing enhanced or inverted NMR lines. This technique is known as "switched external magnetic field chemically induced dynamic nuclear polarization” (SEMF-CIDNP) [68,69].

\section{Diffusion in radical reactions}

The magnitude and features of CIDNP, CIDEP, and chemical MFEs in liquids depend critically on the diffusive motion of the RP involved. This motion is modeled well as occurring in a series of encounters using Noyes' formalism [70]. According to Adrian [71-74], Pedersen, and Freed [17-22,75], the RP undergoes qualitatively different spin evolution depending on the distance between the radicals. During a diffusive encounter, the radicals are in close proximity and $\mathrm{S} \leftrightarrow \mathrm{T}$ inter-conversion is quenched by the strong exchange interaction, that is, by the term $e^{-r / r_{j}} J_{0} \hat{\mathbf{S}}^{\mathrm{A}} \cdot \hat{\mathbf{S}}^{\mathrm{B}}$ in eq. 20. In contrast, during a diffusive excursion, the radicals are separate to distances where the exchange interaction makes negligible contributions to the spin evolution, which proceeds under the influence of the remaining hyperfine and Zeeman interactions. The extent of $\mathrm{S} \leftrightarrow \mathrm{T}$ inter-conversion is expressed simply in terms of the singlet probability

$$
\left\langle\hat{P}^{\mathrm{S}}\right\rangle(\boldsymbol{B}, t)=\operatorname{Tr}\left(\rho(\boldsymbol{B}, t) \hat{P}^{\mathrm{S}}\right)
$$

where $\rho(\boldsymbol{B}, t)$ is the RP density matrix, which evolves according to the Liouville-von Neumann equation, under the influence of the Hamiltonian in eq. 20 and $\hat{P}^{\mathrm{S}}$ is the RP singlet projection operator.

Let us consider a pair of radicals created in close proximity that have subsequently diffused apart. The re-encounter probability distribution $f(t)$ is defined such that $f(t) \mathrm{d} t$ is the probability that they next encounter one another between times $t$ and $t+\mathrm{d} t$. If subsequent re-encounters are important, one may define $f_{n}(t)$ as the probability distribution for $n^{\text {th }}$ re-encounters at time $t$. 
Since on re-encountering, only RPs in a singlet spin state are able to react, the probability of reaction is $\left\langle\hat{P}^{\mathrm{S}}\right\rangle(t) f(t) \mathrm{d} t$. Hence, the yield of singlet product ("singlet yield") from first re-encounters is

$$
\Phi_{\mathrm{S}}(\boldsymbol{B})=\int_{0}^{\infty}\left\langle\hat{P}^{\mathrm{S}}\right\rangle(\boldsymbol{B}, t) f(t) \mathrm{d} t
$$

It is often assumed that after a first re-encounter, radicals either react or diffuse apart forever.

In the analysis of MFEs and other spin chemical effects, two functional forms of $f(t)$ are used widely. The "exponential model" [12,14,76] takes

$$
f(t)=k e^{-k t}
$$

where $k$ is a phenomenological rate constant. The "diffusion model", introduced by Adrian [71-74], assumes that the radicals diffuse in a random flight during each excursion. This assumption gives, after some algebra, a re-encounter probability

$$
f(t)=\frac{R_{\sigma}\left(R_{0}-R_{\sigma}\right)}{R_{0}}\left(\frac{1}{4 \pi D t}\right)^{\frac{3}{2}} \exp \left(\frac{-\left(R_{0}-R_{\sigma}\right)^{2}}{4 D t}\right)
$$

where $R_{\sigma}$ is the distance at which the radicals may react to form products, $R_{0}$ is the separation of the radicals as they leave the strong exchange region, and $D$ is the mutual diffusion coefficient for the two radicals.

These concepts are illustrated in Fig. 6, which summarizes recent efforts to extract an empirical measured re-encounter distribution $f(t)$ from experimental data [53]. MFEs ("MARY spectra") were measured for the photochemically induced reaction between Py and DMA, which proceeds according to Fig. 6a. The applied magnetic field was modulated at an audio frequency and typically swept from -3 to $+23 \mathrm{mT}$ whilst making phase-sensitive measurements of the component of the fluorescence intensity at the modulation frequency. This raw signal is equivalent to the derivative of the field effect $\mathrm{d} \Phi_{\mathrm{S}} / \mathrm{d} B_{0}$, which may be calculated as described above.

Isotopic substitution is a powerful tool for understanding MFEs. In most cases, it causes negligible changes in the structure and reactivity of the molecules in question. Yet, the hyperfine couplings to those nuclei will be altered, since these are proportional to their magnetogyric ratio, which differs between isotopes of an element. Furthermore, isotopes often have different nuclear spin, e.g., H has spin $I=1 / 2$ whilst D has $I=1$. Hence, to gain further information about the Py DMA system, hydrogen atoms on either or both of the radicals were selectively replaced with deuterium. The signals measured from the four isotopomer combinations are shown in Fig. 6c. Calculating the spin evolution in each RP according to the theory above, and using appropriate regularization methods, it was possible to extract empirical re-encounter probabilities $f(t)$ for this system. These are shown in Fig. 6d.

Other workers have studied RP reactions in micelles [77-80] or in more esoteric systems such as carbon nanotubes [81] where the diffusive motion of radicals is quite different to that in isotropic solution. Accurate simulation of such experiments depends on a sound physical model of diffusion and is a good test of the theory. For experiments in strong magnetic fields, Pedersen has introduced a theory based on Green's functions capable of treating diffusion in many different media $[82,83]$.

In some circumstances, it is important to allow connections between the RP spin evolution, diffusion and reaction, e.g., when investigating in detail the effects of exchange and dipolar interactions [51], which vary strongly as a function of inter-radical separation. Hence, at the end of a diffusive excursion, the RP spin state can depend in detail on the path that the radicals took. These effects may be modeled by treating the exchange and dipolar interactions as stochastic perturbations to the RP spin Hamiltonian. Product yields may then be found using a stochastic Liouville equation $[84,85]$, albeit at a cost of more time- and memory-intensive calculation. 


\section{"Rules of thumb" for chemical low-field effects}

In many cases, such a detailed interpretation of observed MFEs is not necessary. Indeed, when the radicals involved are larger or less symmetric, the number of inequivalent nuclear spins that must be included in an exact calculation of the spin evolution grows. The dimensions of the RP density matrix $\rho(\boldsymbol{B}, t)$ grow exponentially with the number of inequivalent nuclear spins. The CPU time and memory required to calculate its time evolution, using the Liouville-von Neumann equation, therefore also increases exponentially.

MARY spectra may instead be characterized qualitatively by the positions of a small number of features, illustrated in Fig. 7a. The figure shows the field dependence of the singlet yield in the simplest possible RP, which has only a single magnetic nucleus $\left({ }^{1} \mathrm{H}\right)$ on one of the radicals and none on the other (a "one proton RP"). The chief features are:

$\Phi_{\mathrm{S}}(\infty)$ : The saturation value of the singlet yield at field strengths above $\sim 100 \mathrm{mT}$. (This neglects the fact that at high field strengths $\sim 1 \mathrm{~T}$, the $\Delta \mathrm{g}$ mechanism becomes operative and the singlet yield will begin to decrease as discussed in connection with Fig. 4.)

$B_{1 / 2}$ : The field strength at which the singlet yield is midway between its zero-field and saturation values.

$B_{\mathrm{LFE}}: \quad$ Often, there is an initial decrease in singlet yield upon application of fields smaller than $1 \mathrm{mT}$, which is known as the low field effect (LFE) [64]. The field at this minimum is termed $B_{\text {LFE }}$.

$\Delta \Phi_{\mathrm{S}}\left(B_{\mathrm{LFE}}\right):$ The change in singlet yield between zero field and the LFE minimum ("LFE depth").

Expressions involving these parameters are used widely in the literature.

Some insight into these MARY spectra may be gained using a semi-classical approximation introduced by Knapp, Schulten, and Wolynes [86,87]. This is derived by assuming that whilst the unpaired electron spins on each radical behave quantum mechanically, the nuclear spins instead behave as classical magnetic dipoles. The unpaired electron spin in each radical is assumed to precess around the resultant of these nuclear dipoles and the applied magnetic field. A result from polymer statistical physics allows integration over all possible orientations of the nuclear spins to give a closed-form expression for the time-dependent singlet probability in any RP (eq. 3.28 in [88]).

Within the semi-classical approximation, RP product yields are determined solely by the effective HFC constants on each radical, defined by

$$
\tilde{a}_{N}^{2}=\frac{4}{3} \sum_{i} a_{N i}^{2} I_{i}\left(I_{i}+1\right)
$$

Importantly, the time taken to evaluate this approximate singlet probability does not depend on the number of magnetic nuclei in the RP, which is in stark contrast with the exponential growth in calculation time for the exact quantum calculation.

Indeed, in the limit of a large number of nuclei at high fields, the saturation value for the singlet yield may be derived quantum mechanically [88]

$$
\Phi_{\mathrm{S}}=\frac{1}{2}+\frac{k_{*} \sqrt{\pi}}{2} \exp \left(k_{*}^{2}\right) \operatorname{erfc}\left(k_{*}\right) \quad \text { where } k_{*}=\frac{k \sqrt{2}}{\tilde{a}_{\mathrm{RP}}}
$$

This depends only on the exponential model rate constant $k$ and the overall RP effective HFC constant

$$
\tilde{a}_{\mathrm{RP}}=\sqrt{\tilde{a}_{\mathrm{A}}^{2}+\tilde{a}_{\mathrm{B}}^{2}}
$$

which is a well-known experimental parameter. 
To test the limits of validity of predictions based on the semi-classical approximation, one can use a Monte Carlo approach described in [53]. Figures 7b and 7c summarize the results from such a calculation using an ensemble of 12737 RPs, each with six HFCs to spin- $1 / 2$ nuclei, three on each radical. These HFCs were drawn randomly such that the effective HFCs on each radical were uniformly distributed between 0 and $1.5 \mathrm{mT}$. After selection of the HFCs, MARY spectra were calculated for each member of the ensemble at 1000 field strengths varying logarithmically between $10^{-11}$ and $10 \mathrm{mT}$ and at zero field. Exponential model rate constants were taken between $10^{-2}$ and $10^{12} \mathrm{~s}^{-1}$, a range which covers the typical experimental values and several orders of magnitude either side. In total, these calculations involved more than 300000000 singlet yield calculations and were performed using the resources of the Oxford Supercomputing Centre. Analysis of these results allows "rules of thumb" to be determined for all the MARY curve parameters described above $[53,88]$. The example in Fig. 7 shows how the depth of the LFE depends on the RP lifetime and HFCs. In Fig. 7b, it is apparent that in longlived RPs, essentially any HFC constants will give rise to a significant LFE. Whereas when the lifetime is that of a typical solution-phase RP, Fig. 7c shows that strong LFEs arise only when one of the radicals has a large effective HFC constant and that of the other is small. This dependence on the RP lifetime explains a long-standing apparent disagreement in the literature between the predictions of [89] and [64].

\section{Optically detected zero-field EPR}

According to eq. 20, time-dependent magnetic fields ought also to affect the yield of RP reactions. For simple organic radicals, the HFC constants are on the order of $1 \mathrm{mT}$. Hence, one would expect to observe resonant responses to fields at frequencies of the order of $30 \mathrm{MHz}$ (the scaling factor is $28 \mathrm{MHz}$ $\mathrm{mT}^{-1}$ ). Such OMFEs have indeed been measured experimentally $[55-57,90]$ by measuring the yield of an RP reaction in the presence of an RF field whose frequency was swept between 5 and $80 \mathrm{MHz}$. These measurements typically produce a spectrum containing two broad resonances, centered around the effective HFC constants of each radical. In principal, this information might be used to identify the radical species involved as intermediates in a complex system.

Yet in practice, the OMFE experiment is quite exacting: the sample must be shielded from the Earth's field using $\mu$-metal sheeting; and the RF circuitry and field coils employed must be capable of being operated over the range $5-80 \mathrm{MHz}$ without introducing power variations, pick-up, or other artefacts. Furthermore, despite all these effects, the observed changes in reaction yield are found to be relatively small; this limits the signal-to-noise ratio of the spectra that can be measured.

The obvious extension from static field effects (MARY spectra) and OMFEs is to apply both static and RF fields simultaneously whilst measuring the reaction yield. This technique of OD-EPR affords many opportunities to investigate the response of RP systems by changing parameters such as the strengths of the static $B_{0}$ and RF $B_{1}$ fields, their relative orientation $\theta$ and the frequency $v_{\mathrm{rf}}$ and polarization of the RF field along with all the other variables familiar from static MFEs, e.g., temperature, solvent viscosity, and the method of excitation. Two such experiments, conducted for radical ion pairs in viscous solvents by Timmel, Hore, and co-workers [63,91-93], are presented in Figs. 8 and 9.

The data in Fig. 8 are typical of those which can be obtained by low-field OD-EPR, showing the good signal-to-noise ratio that can be obtained. From top to bottom, the three rows show the MFEs that arise in the presence of RF fields whose frequency is smaller than, comparable to, and larger than the effective HFCs in the RP. The different lines in each plot are measurements made with a different angle $\theta$ between the static and RF fields. Experimental data are shown on the left, and on the right, simulations made using the $\gamma$-COMPUTE algorithm [63,94]. This algorithm, adapted from solid-state NMR, exploits the periodicity of the Hamiltonian here to perform an efficient calculation of the reaction yield.

In the bottom row, the data broadly follow the selection rules associated with conventional highfield EPR, where only the component of the RF field perpendicular to the static field $\left(\theta=90^{\circ}\right)$ has any effect. The deepest peak here occurs at the electron Zeeman resonance frequency $(65 \mathrm{MHz} / 28 \mathrm{MHz}$ 
$\mathrm{mT}^{-1}=2.3 \mathrm{mT}$ ), with two smaller peaks on either side produced by the hyperfine interactions in the RPs. In contrast, at lower frequencies, there are significant effects whatever the relative orientation of $\mathrm{RF}$ and static fields. The shapes of these spectra are hard to rationalize by simple arguments. However, at all three frequencies, the experimental data are in good qualitative agreement with the predictions of the RPM calculated using the $\gamma$-COMPUTE algorithm.

More recently, experiments in Oxford have extended the OD-EPR technique to vary the polarization of the RF fields that are applied to the reacting sample. An example of the difference between field effects in the presence of linearly or circularly (left/right) polarized RF fields is shown at the topleft of Fig. 9. Once again, simulations made using the $\gamma$-COMPUTE algorithm are able to accurately reproduce the experimental data. The remaining panels in Fig. 9 show simulations made with different values for the RF field strength $B_{1}$. When the effects of all three polarizations are viewed together, the shape of the field effects is remarkably sensitive to the RF field strength [88,91,93]. This makes it possible here to determine the field strength employed experimentally, which is remarkably difficult to determine by other means.

The Earth's magnetic field has a typical strength of $50 \mu \mathrm{T}$, and, therefore, MFEs in nature will operate in this low-field regime. This work is therefore relevant to the debate on the putative adverse health effects of environmental magnetic fields, and also to the fascinating debate on the origins of the magnetic compass sense of migratory birds. Measuring the response of a biological system to combined static and RF fields at several different relative orientations is a good test for the operation of the RPM in vivo. Systems where the RPM is operative should show strong orientation effects as in Fig. 8 whatever the nature of the radicals involved, whereas magnetic effects due to other mechanisms would be very unlikely to show such an effect [92]. Such experiments have recently been performed on European robins, which are able to detect the Earth's magnetic field, supporting the suggestion that this sense is based on RPM chemistry [95].

\section{APPLICATIONS IN THE LIFE SCIENCES}

The chemical effects of magnetic fields have a contribution to make in the life sciences. Two areas of particular interest are the magnetic "compass" sense possessed by many birds and other animals which enables them to use the Earth's magnetic field as a guide during migration, of which more below, and the allegedly harmful effects of weak environmental electromagnetic fields on human health. These include fears that mobile telephones and electricity transmission lines might cause cancer, specifically childhood leukaemia [96-99]. Since spin chemistry provides the only established physicochemical basis for interpreting MFEs, it has an important role in this debate [30,76,100]. Stronger magnetic fields may be used to probe the mechanism of biologically relevant reactions.

\section{Electron transfer}

Many MFEs are known on reactions occurring in solids or the interior of proteins. The mechanism of these reactions revolves around a spin selective reaction step following the scheme in Fig. 2 and is similar in many respects to that of the liquid-phase reactions discussed above. Yet, whereas in liquids, diffusional motion of the radicals plays a central role, in solids and proteins, there is little scope for diffusive motion on the required time scale to produce MFEs. Instead, reaction still proceeds following Fig. 2, but the rate constants $k_{\mathrm{S}}$ and $k_{\mathrm{T}}$ now describe one-electron transfers, whilst $k_{\mathrm{S}}^{\mathrm{b}}$ and $k_{\mathrm{T}}^{\mathrm{b}}$ are the corresponding back-electron transfer rates. The factors determining these rates have been tested extensively in proteins [47] and are accurately described by Marcus theory. In broad terms, the rate of electron transfer between two sites increases as the edge-to-edge separation between them decreases, or as the orbital overlap between them increases or as the Gibbs energy of reaction becomes more well matched. These ideas are illustrated in Fig. 10, which shows the series of sequential electron-transfer 

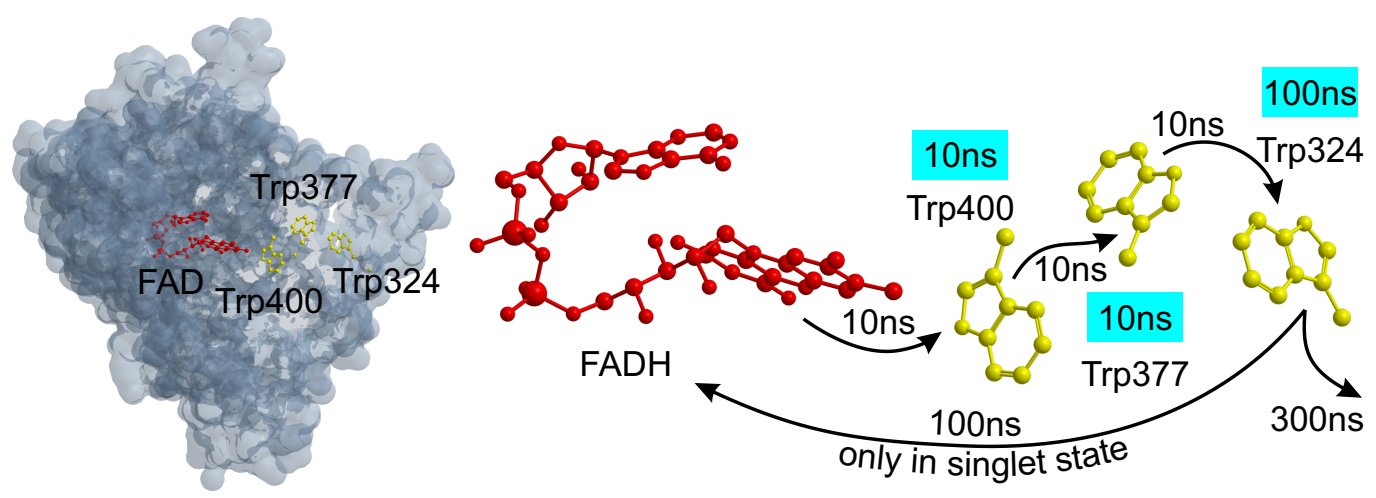

Fig. 10 Cryptochrome (CRY) has been proposed to be responsible for the magnetic field "compass" sensitivity of birds. The photocycle of CRY is as yet unknown. One suggestion [107] is that as light is absorbed, it triggers photochemical electron transfer from the flavin cofactor to Trp 400. The unpaired electron would then continue to "hop" rapidly to Trp 377 and then to Trp 324. At this point, there is a competition between back-electron transfer to the flavin or further reaction leading to generation of a signalling state of CRY. Data from Protein Data Bank file 1U3C. Drawn following [101,102]; see therein for further details.

steps that are thought to produce MFEs in the cryptochrome (CRY) family of proteins. The distances between the electron sites favor a rapid sequential electron transfer [101].

In theoretical work, it is often assumed for simplicity that the decay of singlet and triplet RPs is equal with the triplet RPs undergoing further electron transfer and the singlet ones undergoing backelectron transfer at the same rate, i.e., $k_{\mathrm{T}}=k_{\mathrm{S}}^{\mathrm{b}}=k$ and $k_{\mathrm{S}}=k_{\mathrm{T}}^{\mathrm{b}}=0$. With this assumption of first-order kinetics, the resulting expressions for product yield are the same as those for liquid-phase reactions. However, it is important to remember that whereas in the liquid phase, $k$ governs the probability distribution of re-encounters between a diffusing RP within the exponential model: $f(t)=k e^{-k t}$, in the solid state $k$ is a true first-order rate constant.

The MFEs on protein electron transfer that have been most extensively studied experimentally are those that occur in the reaction centers of photosynthetic bacteria after they have been chemically modified to impede forward electron transfer down one of the two almost symmetrical branches of cofactors in the photosynthetic reaction center. MFEs and RYDMR spectra have been measured at room temperature in solution for fields as low as a few $\mathrm{mT}$ [103-106].

MFEs have also been measured in enzymes that react via RP intermediates such as coenzyme $B_{12}$ [29], photolyase [107], and horseradish peroxidase [108], although this has recently attracted controversy [109-111]. See [29] for a review of other MFEs in biology.

\section{Magnetic isotope effects}

Reactions occurring by the RPM are susceptible not only to external magnetic fields, but also to changes in the intramolecular magnetic fields arising from HFCs between the unpaired electrons and magnetic nuclei. These effects may be exploited to obtain mechanistic insight by selective substitution of particular atoms with other isotopes of the same element. This technique was employed in Fig. 6, where hydrogen atoms in the RP were selectively replaced with deuterium, as described above.

The same idea applies to other elements. For example, Buchachenko and co-workers have recently reported [112,113] substantial magnetic isotope effects on the rate of enzymatic ATP production in the presence of ${ }^{24} \mathrm{Mg}(I=0),{ }^{25} \mathrm{Mg}(I=5 / 2)$, and ${ }^{26} \mathrm{Mg}(I=0)$. In ATP-synthase, phosphocreatine, and phosphoglycerate kinases, the rate of reaction was reported to be enhanced by a factor of 2-3 with ${ }^{25} \mathrm{Mg}$ compared to the other isotopes. This magnetic isotope effect is exciting because it is a large ef- 
fect and because the enzymes involved are hugely important in human biochemistry, providing of course that it can be confirmed independently.

\section{Anisotropic effects for an RPM-based compass}

Behavioral studies conducted over several decades in many laboratories show that animals from all five classes of vertebrate and several invertebrates possess a magnetic compass sense [114,115]. However, the physiological basis of magnetoreception remains an enigma. In birds, magnetoreception is known to require ambient light in the higher-energy blue-green region of the spectrum. This is suggestive of a photochemically driven electron transfer. Furthermore, magnetoreception is disrupted by covering the bird's eyes, showing that the source of RPs is located therein. Following this line of thinking, Ritz and Schulten [116] proposed that avian magnetoreception is founded on a photochemical reaction occurring between oriented molecules fixed within the retina as illustrated in Fig. 11.

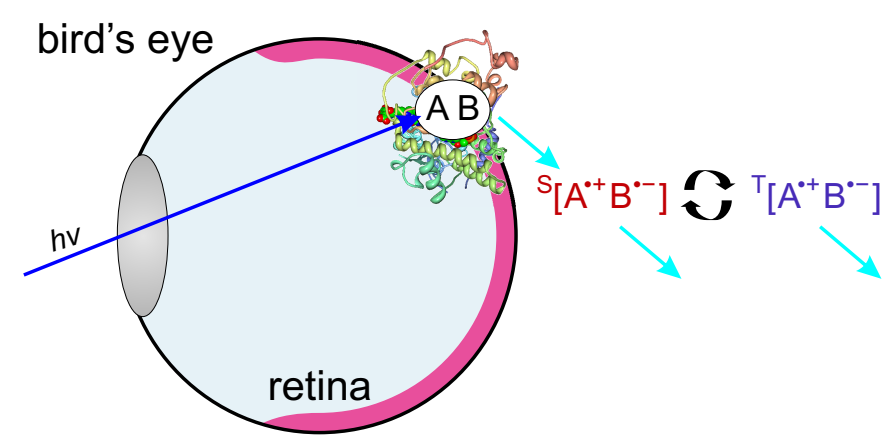

Fig. 11 Schematic model of RPM-based magnetoreception in a bird's eye. After [116].

Radicals in the solid state may be assumed to be immobile. They undergo no significant diffusive translational motion, nor are they able to rotate. Whereas in the liquid phase, diffusive motion of the radicals causes anisotropic hyperfine and dipolar interactions to be averaged away, in the solid state it is quite possible for RPM reaction yields in an oriented sample to depend on the relative alignment of the magnetic field with respect to the sample, which opens the possibility of forming an RPM-based compass [4].

The identity of the RP involved in magnetoreception remains unknown, but Ritz and co-workers named the retinal CRY proteins [116-118] as promising candidates. CRYs are closely related to DNA photolyases, which form an unusually long-lived RP intermediate [119] whose yield varies in an applied magnetic field [107]. The physical chemistry of magnetoreception has been reviewed elsewhere in detail by the author [36].

\section{CONCLUSIONS}

MFEs on yields and lifetimes of chemical systems are widespread. They may be interpreted convincingly by means of the RPM, giving insights into the kinetics, diffusion, and structure of the radicals involved. When static and RF fields are applied simultaneously, chemical MFEs are closely related to more conventional forms of EPR spectroscopy. At this time, the RPM provides the only sound mechanistic basis for interpreting MFEs in biochemistry. 


\section{ACKNOWLEDGMENTS}

I would like to thank: IUPAC for giving me the opportunity to present this work; my former supervisors Prof. P. J. Hore and Dr. C. R. Timmel for their invaluable advice and encouragement over the years; as well as Somerville and Merton College Oxford for Junior Research Fellowships. I thank Prof. P. J. Hore, Mr. C. J. Wedge, and Dr. C. J. Rodgers for helpful comments. I also thank my fellow students, friends, and colleagues who made my time as a graduate student so exciting.

\section{REFERENCES}

1. U. E. Steiner, T. Ulrich. Chem. Rev. 89, 51 (1989).

2. H. Hayashi. Introduction to Dynamic Spin Chemistry: Magnetic Field Effects on Chemical and Biochemical Reactions, World Scientific, Singapore (2004).

3. F. Z. Tang, A. Katsuki, Y. Tanimoto. Mol. Phys. 104, 1667 (2006).

4. K. Maeda, K. B. Henbest, F. Cintolesi, I. Kuprov, C. T. Rodgers, P. A. Liddell, D. Gust, C. R. Timmel, P. J. Hore. Nature 453, 387 (2008).

5. H. Hayashi. RIKEN Rev. 44, 7 (2002).

6. R. C. Johnson, R. E. Merrifield, P. Avakian, R. B. Flippen. Phys. Rev. Lett. 19, 285 (1967).

7. R. E. Merrifield. J. Chem. Phys. 48, 4318 (1968).

8. B. Brocklehurst. Nature 221, 921 (1969).

9. B. Brocklehurst. J. Chem. Soc., Faraday Trans. 72, 1869 (1976).

10. B. Brocklehurst. Faraday Discuss. Chem. Soc. 63, 96 (1977).

11. J. Bargon. Helv. Chim. Acta 89, 2082 (2006).

12. R. Kaptein, J. L. Oosterhoff. Chem. Phys. Lett. 4, 195 (1969).

13. G. L. Closs. J. Am. Chem. Soc. 91, 4552 (1969).

14. R. G. Lawler, G. T. Evans. Ind. Chim. Belge 36, 1087 (1971).

15. S. K. Wong, J. K. S. Wan. J. Am. Chem. Soc. 94, 7197 (1972).

16. P. W. Atkins, I. C. Buchanan, R. C. Gurd, K. A. McLauchlan, A. F. Simpson. J. Chem. Soc. D: Chem. Commun. 9, 513 (1970).

17. J. B. Pedersen, J. H. Freed. J. Chem. Phys. 57, 1004 (1972).

18. J. B. Pedersen, J. H. Freed. J. Chem. Phys. 58, 2746 (1973).

19. J. B. Pedersen, J. H. Freed. J. Chem. Phys. 59, 2869 (1973).

20. J. B. Pedersen, J. H. Freed. J. Chem. Phys. 61, 1517 (1974).

21. J. B. Pedersen, J. H. Freed. J. Chem. Phys. 62, 1790 (1975).

22. J. B. Pedersen, J. H. Freed. J. Chem. Phys. 62, 1706 (1975).

23. K. Schulten, H. Staerk, A. Weller, H.-J. Werner, B. Nickel. Z. Phys. Chem. 101, 371 (1976).

24. Z. Schulten, K. Schulten. J. Chem. Phys. 66, 4616 (1977).

25. M. Goez. Concepts Magn. Reson. 7, 69 (1995).

26. P. J. Hore, C. G. Joslin, K. A. Mclauchlan. Chem. Soc. Rev. 8, 29 (1979).

27. E. L. Frankevich, S. I. Kubarev. In Triplet State ODMR Spectroscopy: Techniques and Applications to Biophysical Systems, pp. 137-183, Wiley-Interscience, New York (1982).

28. J. R. Woodward. Prog. React. Kinet. Mech. 27, 165 (2002).

29. C. B. Grissom. Chem. Rev. 95, 3 (1995).

30. B. Brocklehurst. Chem. Soc. Rev. 31, 301 (2002).

31. H. Murai. J. Photochem. Photobiol., C 3, 183 (2003).

32. C. R. Timmel, K. B. Henbest. Philos. Trans. R. Soc. London, Ser. A 362, 2573 (2004).

33. A. L. Buchachenko, E. L. Frankevich. Chemical Generation and Reception of Radio- and Microwaves, VCH, Weinheim (1994).

34. S. Nagakura, H. Hayashi, T. Azumi. Dynamic Spin Chemistry: Magnetic Controls and Spin Dynamics of Chemical Reactions, John Wiley, New York (1998). 
35. K. M. Salikhov, Y. N. Molin, R. Z. Sagdeev, A. L. Buchachenko. Spin Polarization and Magnetic Effects in Radical Reactions, Elsevier, Amsterdam (1984).

36. C. T. Rodgers, P. J. Hore. Proc. Natl. Acad. Sci. USA (2009). In press.

37. K. A. McLauchlan, U. E. Steiner. Mol. Phys. 73, 241 (1991).

38. M. H. L. Pryce. Proc. Phys. Soc. A 63, 25 (1950).

39. A. Abragam, M. H. L. Pryce. Proc. R. Soc. London, Ser. A 205, 135 (1951).

40. M. H. Levitt. Spin Dynamics: Basics of Nuclear Magnetic Resonance, John Wiley, New York (2001).

41. P. W. Atkins, R. S. Friedman. Molecular Quantum Mechanics, Oxford Unversity Press, Oxford (1997).

42. N. M. Atherton. Electron Spin Resonance, Ellis Horwood, Chichester (1973).

43. F. Neese. J. Chem. Phys. 115, 11080 (2001).

44. R. Improta, V. Barone. Chem. Rev. 104, 1231 (2004).

45. N. Rega, M. Cossi, V. Barone. J. Chem. Phys. 105, 11060 (1996).

46. F. Illas, I. D. R. Moreira, C. de Graaf, V. Barone. Theor. Chem. Acc. 104, 265 (2000).

47. C. C. Moser, J. M. Keske, K. Warncke, R. S. Farid, P. L. Dutton. Nature 355, 796 (1992).

48. J. Abe, T. Sano, M. Kawano, Y. Ohashi, M. M. Matsushita, T. Iyoda. Angew. Chem. 113, 600 (2001).

49. L. E. Sinks, E. A. Weiss, J. M. Giaimo, M. R. Wasielewski. Chem. Phys. Lett. 404, 244 (2005).

50. R. R. Ernst, G. Bodenhausen, A. Wokaun. Principles of Nuclear Magnetic Resonance in One and Two Dimensions, Oxford University Press, Oxford (1988).

51. A. R. O'Dea, A. F. Curtis, N. J. B. Green, C. R. Timmel, P. J. Hore. J. Phys. Chem. A 109, 86973 (2005).

52. O. Efimova, P. J. Hore. Biophys. J. 94, 1565 (2008).

53. C. T. Rodgers, S. A. Norman, K. B. Henbest, C. R. Timmel, P. J. Hore. J. Am. Chem. Soc. 129, 6746 (2007).

54. W. Lersch, M. E. Michel-Beyerle. Chem. Phys. 78, 115 (1983).

55. J. R. Woodward, R. J. Jackson, C. R. Timmel, P. J. Hore, K. A. McLauchlan. Chem. Phys. Lett. 272, 376 (1997).

56. D. V. Stass, J. R. Woodward, C. R. Timmel, P. J. Hore, K. A. McLauchlan. Chem. Phys. Lett. 329, 15 (2000).

57. C. R. Timmel, P. J. Hore. Chem. Phys. Lett. 257, 401 (1996).

58. A. D. Trifunac, J. P. Smith. Chem. Phys. Lett. 73, 94 (1980).

59. V. A. Morozov, O. N. Antzutkin, A. V. Koptyug, A. B. Doktorov. Mol. Phys. 73, 517 (1991).

60. V. A. Morozov, A. B. Doktorov. Chem. Phys. 153, 313 (1991).

61. V. A. Morozov, A. B. Doktorov. Chem. Phys. 153, 333 (1991).

62. F. B. Sviridenko, D. V. Stass, T. V. Kobzeva, E. V. Tretyakov, S. V. Klyatskaya, E. V. Mshvidobadze, S. F. Vasilevsky, Y. N. Molin. J. Am. Chem. Soc. 126, 2807 (2004).

63. C. T. Rodgers, K. B. Henbest, P. Kukura, C. R. Timmel, P. J. Hore. J. Phys. Chem. A 109, 5035 (2005).

64. C. R. Timmel, U. Till, B. Brocklehurst, K. A. McLauchlan, P. J. Hore. Mol. Phys. 95, 71 (1998).

65. M. Liedvogel, K. Maeda, K. Henbest, E. Schleicher, T. Simon, C. R. Timmel, P. J. Hore, H. Mouritsen. PLoS ONE 2 (2007).

66. E. V. Kalneus, A. A. Kipriyanov, P. A. Purtov, D. V. Stass, Y. N. Molin. Dokl. Phys. Chem. 415, 170 (2007).

67. H. Murai, S. Tero-Kubota, S. Yamauchi. In Electron Paramagnetic Resonance, Vol. 17, pp. 130-163, The Royal Society of Chemistry, Cambridge (2000).

68. S. R. Shakirov, T. N. Makarov, E. G. Bagryanskaya, R. Z. Sagdeev. Phys. Chem. Chem. Phys. 3, $3672(2001)$. 
69. T. N. Makarov, E. G. Bagryanskaya, S. R. Shakirov, N. N. Lukzen, R. Z. Sagdeev. Chem. Phys. Lett. 317, 252 (2000).

70. R. M. Noyes. J. Chem. Phys. 22, 1349 (1954).

71. F. J. Adrian. J. Chem. Phys. 53, 3374 (1970).

72. F. J. Adrian. J. Chem. Phys. 54, 3912 (1971).

73. F. J. Adrian. J. Chem. Phys. 54, 3918 (1971).

74. F. J. Adrian. In Chemically Induced Magnetic Polarisation: Proceedings of the NATO Advanced Study Institute held at Sogesta, Urbino, Italy, pp. 77-105, D. Reidel, Dordrecht (1977).

75. J. B. Pedersen. In Spin Chemistry: Spin Polarization and Magnetic Field Effects in Photochemical Reactions, pp. 71-89, The Oji International Conference on Spin Chemistry (1991).

76. B. Brocklehurst, K. A. McLauchlan. Int. J. Radiat. Biol. 69, 3 (1996).

77. S. D. Choudhury, S. Basu. J. Phys. Chem. A 109, 8113 (2005).

78. A. A. Neufeld, M. J. Hansen, J. B. Pedersen. Chem. Phys. 278, 129 (2002).

79. T. Miura, H. Murai. J. Phys. Chem. A 112, 2526 (2008).

80. K. Maeda, T. Miura, T. Arai. Mol. Phys. 104, 1779 (2006).

81. N. N. Lukzen, K. L. Ivanov, V. A. Morozov, R. Z. Sagdeev, D. Kattnig, G. Grampp. Dokl. Phys. Chem. 409, 233 (2006).

82. M. J. Hansen, A. A. Neufeld, J. B. Pedersen. Chem. Phys. 260, 125 (2000).

83. A. B. Doktorov, J. B. Pedersen. Chem. Phys. 322, 433 (2006).

84. R. Kubo. J. Math. Phys. 4, 174 (1963).

85. D. Gamliel, H. Levanon. Stochastic Processes in Magnetic Resonance, World Scientific, Singapore (1995).

86. K. Schulten, P. G. Wolynes. J. Chem. Phys. 68, 3292 (1978).

87. E. W. Knapp, K. Schulten. J. Chem. Phys. 71, 1878 (1979).

88. C. T. Rodgers. "Magnetic field effects in chemical systems", DPhil thesis, University of Oxford (2007).

89. D. V. Stass, N. N. Lukzen, B. M. Tadjikov, Y. N. Molin. Chem. Phys. Lett. 233, 444 (1995).

90. R. J. Jackson, K. A. McLauchlan, J. R. Woodward. Chem. Phys. Lett. 236, 395 (1995).

91. S. A. Norman. "Magnetic field effects on radical pair reactions", DPhil thesis, University of Oxford (2006).

92. K. B. Henbest, P. Kukura, C. T. Rodgers, P. J. Hore, C. R. Timmel. J. Am. Chem. Soc. 126, 8102 (2004).

93. C. J. Wedge. "Magnetic field effects on radical recombination reactions", MChem thesis, University of Oxford (2005).

94. M. Hohwy, H. Bildsoe, H. J. Jakobsen, N. C. Nielsen. J. Magn. Reson. 136, 6 (1999).

95. T. Ritz, P. J. Hore, C. R. Timmel, C. T. Rodgers, K. Stapput, P. Thalau, R. Wiltschko, W. Wiltschko. Biophys. J. (2009). In press.

96. R. Doll. National Radiological Protection Board (2001).

97. M. Havas. Environ. Rev. 8, 173 (2000).

98. UK Childhood Cancer Study Investigators. Lancet 354, 1925 (1999).

99. S. W. Stewart. Independent Expert Group on Mobile Phones (2000).

100. K. McLauchlan. Phys. World 41 (1992).

101. I. A. Solov'yov, D. E. Chandler, K. Schulten. Biophys. J. 92, 2711 (2007).

102. S. Johnsen, K. J. Lohmann. Phys. Today 61, 29 (2008).

103. M. Volk, G. Aumeier, T. Langenbacher, R. Feick, A. Ogrodnik, M.-E. Michel-Beyerle. J. Phys. Chem. B 102, 735 (1998).

104. C. E. D. Chidsey, L. Takiff, R. A. Goldstein, S. G. Boxer. Proc. Natl. Acad. Sci. USA 82, 6850 (1985).

105. Y. Liu, R. Edge, K. Henbest, C. R. Timmel, P. J. Hore, P. Gast. Chem. Commun. 2, 174 (2005).

106. A. J. Hoff, J. Deisenhofer. Phys. Rep. 287, 2 (1997). 
107. K. B. Henbest, K. Maeda, P. J. Hore, M. Joshi, A. Bacher, R. Bittl, S. Weber, C. R. Timmel, E. Schleicher. Proc. Natl. Acad. Sci. USA 105, 14395 (2008).

108. M. B. Taraban, T. V. Leshina, M. A. Anderson, C. B. Grissom. J. Am. Chem. Soc. 119, 5768 (1997).

109. A. R. Jones, S. Hay, J. R. Woodward, N. S. Scrutton. J. Am. Chem. Soc. 129, 15718 (2007).

110. M. S. Afanasyeva, M. B. Taraban, P. A. Purtov, T. V. Leshina, C. B. Grissom. J. Am. Chem. Soc. 128, 8651 (2006).

111. A. R. Jones, N. S. Scrutton, J. R. Woodward. J. Am. Chem. Soc. 128, 8408 (2006).

112. A. L. Buchachenko, D. A. Kouznetsov, M. A. Orlova, A. A. Markarian. Proc. Natl. Acad. Sci. USA 102, 10793 (2005).

113. A. L. Buchachenko, D. A. Kouznetsov, N. N. Breslavskaya, M. A. Orlova. J. Phys. Chem. B 112, 2548 (2008).

114. S. Johnsen, K. J. Lohmann. Nat. Rev. Neurosci. 6, 703 (2005).

115. R. Wiltschko, W. Wiltschko. Magnetic Orientation in Animals, Springer, Berlin (1995).

116. T. Ritz, S. Adem, K. Schulten. Biophys. J. 78, 707 (2000).

117. A. Sancar. Chem. Rev. 103, 2203 (2003).

118. C. L. Partch, A. Sancar. Photochem. Photobiol. 81, 1291 (2005).

119. S. Weber. Biochim. Biophys. Acta 1707, 1 (2005). 